\title{
Self-Similar Perturbation of an Accretion Disc around Merging Black Holes
}

\author{
A. G. Zhilkin ${ }^{a}$ * and D. V. Bisikalo ${ }^{a}$ \\ ${ }^{a}$ Institute of Astronomy, Russian Academy of Sciences, Moscow, 119017 Russia \\ *e-mail: zhilkin@inasan.ru \\ Received June 7, 2021; revised July 26, 2021; accepted July 27, 2021
}

\begin{abstract}
We study the possibility that merging of a binary black hole surrounded by a circumbinary accretion disc may produce an electromagnetic response. When black holes are merging, the mass of a target object decreases due to radiating gravitational waves; and, consequently, the disc experiences perturbations, which manifest themselves in, among other phenomena, producing rather intense shock waves. Based on the numerical modeling results, we analyze the self-similar solution that describes the evolution of a perturbed accretion disc while accounting for the vertical expansion effect leading to the adiabatic cooling of matter heated by shock waves. The methodology developed allows us to correctly estimate the magnitude of the heating of matter caused by shock waves and, consequently, to determine parameters of the electromagnetic radiation produced by the disc, including the light curve, the radiation spectrum, and the outburst duration.
\end{abstract}

Keywords: gravitational waves, black holes, binary stars, accretion discs

DOI: $10.1134 / \mathrm{S} 1063772921110081$

\section{INTRODUCTION}

Since 2015, when gravitational waves were discovered in experiments [1], a new page in astronomy has been turned. A potential of the multi-messenger approach makes it possible to reach a deeper understanding on the nature of astrophysical objects. According to theoretical conceptions [2, 3], binary back holes exhibit the highest intensity of the gravitational-wave radiation [4], while binary back holes of stellar masses may be detected with the highest probability [5,6]. As a matter of fact, starting from 2015, the Laser Interferometer Gravitational-Wave Observatory (LIGO) and the Virgo detectors have registered several gravitational-wave events accompanying the merging of binary black holes [1,7-11]. The question arises: how can we apply the multi-messenger approach to these events, or, in other words, does an electromagnetic response appear, when black holes are merging, and can we observe it?

In the studies of black holes, it is often assumed that these objects are surrounded by accretion discs. The scenarios of forming a disc around a binary supermassive black hole, as well as a binary black hole with a stellar mass, were described in our papers $[12,13]$. Let us consider the problem on perturbation of an accretion disc or an enclosure around merging black holes. According to the general theory of relativity, the energy loss of a binary hole due to radiating gravitational waves results in the formation of a target object with the mass several percent lower than the initial one
[3]. Moreover, due to the momentum loss caused by the deviation from symmetry, the object may get a kick [14] and, consequently, acquire a velocity up to $1000 \mathrm{~km} / \mathrm{s}$ [15]. The matter, surrounding the merging black holes, is perturbed [16], which may result in the luminosity enhancement and quasi-periodic outbursts. The recoil effect of a black hole and the subsequent gravitational perturbation may induce gravitational waves in the accretion disc [17], while the metric perturbations themselves induce mechanical stresses in the disc. They may dissipate over a viscous timescale [18] or may be transformed to shock waves [19]. The disc matter heating induced by shock waves should lead to a sharp surge in the disc luminosity, which can be considered as an electromagnetic response of the system to the merging of a binary black hole.

For the case of merging supermassive black holes in galactic nuclei with a total mass of $10^{6} M_{\odot}$, the $5 \%$ mass loss induces an increase in the luminosity of the accretion disc up to $10^{43} \mathrm{erg} / \mathrm{s}$ [20]. The recoil effect may result in the similar increase of the luminosity; however, its magnitude substantially depends on the poorly determined parameters of a merging binary black hole [21, 22]. The response of approximately the same magnitude could also be expected from black holes of stellar masses. In a paper [23], it was shown that the luminosity of an accretion disc may grow up to $10^{42}-10^{43} \mathrm{erg} / \mathrm{s}$; and the radiation energy will be maximal in the X-ray spectral range even for density 
Table 1. Parameters of the merging binary black holes according to the data of gravitational-wave observations

\begin{tabular}{c|l|c|c|c|c}
\hline Object & $M_{1} / M_{\odot}$ & $M_{2} / M_{\odot}$ & $\Delta M /\left(M_{1}+M_{2}\right), \%$ & $D$, Mpc & Reference \\
\hline GW150914 & $36_{-4}^{+5}$ & $29_{-4}^{+4}$ & $3.3-5.2$ & $410_{-180}^{+160}$ & {$[1]$} \\
GW151226 & $14.2_{-3.7}^{+8.3}$ & $7.5_{-2.3}^{+2.3}$ & $2.9-5.4$ & $440_{-190}^{+180}$ & {$[7]$} \\
GW170104 & $31.2_{-6.0}^{+8.4}$ & $19.4_{-5.9}^{+8.3}$ & $2.3-5.7$ & $880_{-390}^{+450}$ & {$[8]$} \\
GW170608 & $12_{-2}^{+7}$ & $7_{-2}^{+2}$ & $2.8-5.1$ & $340_{-140}^{+140}$ & {$[9]$} \\
GW170814 & $30.5_{-3.0}^{+5.7}$ & $25.3_{-4.2}^{+2.8}$ & $4.0-5.8$ & $540_{-10}^{+130}$ & {$[10]$} \\
\hline
\end{tabular}

The columns (from left to right) contain the object name, the masses of the components, the relative mass change, the distance to the object, and the reference.

waves rather than shock ones. In our papers [12, 24], for the models, the parameters of which corresponded to event GW170814 [10], we calculated the bolometric light curves and electromagnetic spectra and estimated the burst duration. As turned out, a main portion the electromagnetic radiation energy is emitted in the X-ray and gamma ranges; and outbursts of this kind can be registered by the currently available instruments.

The analysis of the results of numerical calculations performed in [12, 24] allowed us to conclude that, in the course of time, under a certain behavior of the density and temperature distributions in an initial nonperturbed disc, the propagation mode of shock waves in a perturbed disc becomes self-similar. Specifically, for the time $t$, the shock wavefront passes the distance described by a power law of $t^{2 / 3}$, while its velocity diminishes with time as $t^{-1 / 3}$. In a paper [13], we obtained a complete self-similar solution of this problem with using the required information about shock waves taken from numerical calculations. From the self-similar solution, one may derive the algebraic integrals for the angular momentum and adiabaticity, as well as find the asymptotic relationships for the selfsimilar functions. With this analytical model, on the basis of the self-similar solution, one may approximately estimate the magnitude of the electromagnetic response of the gravitational-wave event without timeconsuming numerical calculations. In the present paper, we specify more accurately the previous model by accounting for the vertical expansion of a disc, which leads to the adiabatic cooling of matter behind shock waves. The applied technique allows us to make the model closer to the real physical conditions and to estimate reliably the electromagnetic response from merging black holes with stellar masses.

A layout of the paper is as follows. In Section 2, we formulate the problem and describe the main equations. In Section 3, the self-similar equations are presented and analyzed. In Section 4, the calculation results are described. In Section 5, the electromagnetic response characteristics are discussed. Section 6 contains the main conclusions. In the Appendix, we describe some details of the numerical method and the analytical solution of the problem.

\section{MAIN EQUATIONS}

With the Laser Interferometer Gravitational-Wave Observatory (LIGO) and Virgo detectors, two complete series of observations, O1 (from September 12, 2015 to January 19, 2016) and O2 (from November 30, 2016 to August 26, 2017), were carried out. The third series O3 started on April 1, 2019; and it was to be completed on April 30, 2020. However, in late March of 2020 , the observations were stopped due to the coronavirus pandemic. In the complete observational series $\mathrm{O} 1$ and $\mathrm{O} 2$, ten events induced by merging binary black holes were found [11]; among them, only five events have no artifacts. These events are presented in Table 1 together with the estimates for the masses of components, the lost mass portion, and the distance to the corresponding object. As is seen from this table, all of these objects correspond to black holes of stellar masses. In addition, a portion of the mass lost due to merging black holes amounts to $\sim(2-6) \%$. In this paper, we analyze event GW170814, which corresponds to merging the binary black hole with components $30.5 M_{\odot}$ and $25.3 M_{\odot}$ in mass. More specific, we will assume that the mass loss in this event was $5 \%$.

In the studies of black holes, it is often assumed that they are surrounded by accretion discs. The inner radius of an accretion disc around an individual black hole is $3 R_{\mathrm{g}}$, where $R_{\mathrm{g}}=2 G M / c^{2}, G$ is the gravitational constant, and $c$ is the velocity of light. Within this zone, there are no stable circular orbits [3]. Let us assume that a binary black hole is also surrounded by a circumbinary accretion disc. A terminal stage of the merging of black holes moves very quickly, and it takes a fraction of a second. A swift process of merging black holes starts when the distance between them is $A=3 R_{\mathrm{g}, 1}+3 R_{\mathrm{g}, 2}=3 R_{\mathrm{g}}$, where $R_{\mathrm{g}, 1}$ and $R_{\mathrm{g}, 2}$ are the gravitational radii of the components and $R_{\mathrm{g}}$ is the gravitational radius calculated from their total mass $M=M_{1}+M_{2}$ (i.e., the gravitational radius of the accretor) [25-28]. The inner radius of the circumbinary accretion disc is roughly twice the center-to-center spacing of the black holes. This follows from the estimates for the stability of orbits relative to the tidal forces [12, 13] and well agrees with the results of numerical modeling of binary systems with circular 
orbits of the components, the mass ratio of which is close to unity [29-32].

Until the merging moment, the black holes approach each other slowly; and, consequently, due to action of viscous forces, the accretion disc has time to adapt itself to changing the distance between the components. Therefore, we may consider the inner radius of the disc $r_{\mathrm{d}}$ to be approximately six times as large as the gravitational radius of the accretor $R_{\mathrm{g}}$ immediately before the black hole merging. This allows us to ignore the relativistic effects and use the theory by Shakura and Sunyaev [33] when describing the properties of an accretion disc of this kind. In this model, the accretion rate $\dot{M}$ is determined by the dissipation rate of the angular momentum originating from the turbulent viscosity. The turbulence intensity is described by the dimensionless parameter $\alpha$, the value of which does not exceed unity. The critical accretion rate is determined by the Eddington luminosity magnitude

$$
\dot{M}_{\mathrm{cr}}=1.8 \times 10^{-9} \frac{\mathrm{m}}{\chi} \frac{M_{\odot}}{\mathrm{year}},
$$

where $m=M / M_{\odot}$ is the dimensionless mass of the central object. The parameter $\chi$ determines the accretion effectiveness and amounts to $\sim(0.06-0.08)$ for an individual nonrotating black hole [34]. While considering the rotation, the stationary accretion effectiveness grows to $0.32[35,36]$. In the episodic accretion scenario, the effectiveness may reach 0.43 [37]. However, in our model, a particular value of this parameter is not important, since it is the magnitude of the dimensionless accretion rate $\dot{m}=\dot{M} / \dot{M}_{\text {cr }}$ that plays a key role.

According to the model [33], three characteristic zones (A, B, and $\mathrm{C}$ ) are isolated in an accretion disc. Within these zones, the distribution of hydrodynamic quantities is determined by the relative contribution of the gas pressure and the radiation pressure, as well as by the opacity mechanism. In zone $\mathrm{A}$, the radiation pressure dominates, while in zones $\mathrm{B}$ and $\mathrm{C}$, it can be considered negligible as compared to the gas pressure. In zones $\mathrm{A}$ and $\mathrm{B}$, the opacity is determined by the Thomson scattering, while in zone $\mathrm{C}$, the opacity is provided by free-free transitions. In these zones, the vertical structure of the disc differs rather greatly; however, the radial dependences of the density and the temperature turn out to be close to power laws with almost the same indexes.

In this paper, we will consider only zone B [13]. Thus, to describe the density and temperature profiles in an unperturbed accretion disc, the following relationships can be used:

$$
\rho=7.03 \alpha^{-7 / 10} m^{-7 / 10} \dot{m}^{2 / 5}\left(\frac{3 R_{\mathrm{g}}}{r}\right)^{33 / 20} \mathrm{~g} / \mathrm{cm}^{3},
$$

$$
T=3.1 \times 10^{8} \alpha^{-1 / 5} m^{-1 / 5} \dot{m}^{2 / 5}\left(\frac{3 R_{\mathrm{g}}}{r}\right)^{9 / 10} \mathrm{~K},
$$

where the parameter $\alpha$ determines the efficiency of the angular momentum transfer in the disc (the Shakura-Sunyaev parameter).

Consequently, in our model, to specify the initial conditions, we use the following power distributions for the density and the temperature in an unperturbed disc:

$$
\begin{aligned}
& \rho_{0}(r)=\rho_{*}\left(\frac{r}{r_{*}}\right)^{-k_{\mathrm{d}}}, \\
& T_{0}(r)=T_{*}\left(\frac{r}{r_{*}}\right)^{-k_{\mathrm{t}}},
\end{aligned}
$$

where $\rho_{*}$ and $T_{*}$ are the corresponding values at the point $r=r_{*}$. As follows from expressions (2) and (3), for zone $\mathrm{B}$, the indexes are $k_{\mathrm{d}}=33 / 20$ and $k_{\mathrm{t}}=9 / 10$. The initial profiles of the other quantities will be presented below. The Shakura-Sunyaev model [33] is used only to describe the unperturbed state of an accretion disc. After merging the black holes and losing the mass of the central object, the relaxation process in the disc moves relatively quickly, and the subsequent evolution of the disc can be modeled under the approximation of dissipation-free gravitational gas dynamics (i.e., with the viscosity ignored) [12, 24].

The gravitational gas dynamics equations describing the evolution of a geometrically thin accretion disc after merging black holes can be written in the following form for the axially symmetric case in the cylindrical coordinates $(r, \varphi, z)$ :

$$
\begin{gathered}
\frac{\partial \rho}{\partial t}+v_{r} \frac{\partial \rho}{\partial r}+v_{z} \frac{\partial \rho}{\partial z}+\rho\left[\frac{1}{r} \frac{\partial}{\partial r}\left(r v_{r}\right)+\frac{\partial v_{z}}{\partial z}\right]=0 \\
\frac{\partial v_{r}}{\partial t}+v_{r} \frac{\partial v_{r}}{\partial r}+v_{z} \frac{\partial v_{r}}{\partial z}-\frac{v_{\varphi}^{2}}{r}+\frac{1}{\rho} \frac{\partial P}{\partial r} \\
+(1-\xi) \frac{G M}{r^{2}}=0 \\
\frac{\partial v_{z}}{\partial t}+v_{r} \frac{\partial v_{z}}{\partial r}+v_{z} \frac{\partial v_{z}}{\partial z}+\frac{1}{\rho} \frac{\partial P}{\partial z}+(1-\xi) \frac{G M}{r^{3}} z=0, \\
\frac{\partial v_{\varphi}}{\partial t}+v_{r} \frac{\partial v_{\varphi}}{\partial r}+v_{z} \frac{\partial v_{\varphi}}{\partial z}+\frac{V_{r} v_{\varphi}}{r}=0 \\
\frac{\partial \varepsilon}{\partial t}+v_{r} \frac{\partial \varepsilon}{\partial r}+v_{z} \frac{\partial \varepsilon}{\partial z}+\frac{P}{\rho}\left[\frac{1}{r} \frac{\partial}{\partial r}\left(r v_{r}\right)+\frac{\partial v_{z}}{\partial z}\right]=0 .
\end{gathered}
$$

The above equations contain the following quantities: the density $\rho$; the radial, vertical, and azimuthal velocities, $v_{r}, v_{z}$, and $v_{\varphi}$, respectively; the pressure $P$; the specific internal energy $\varepsilon$, the total mass $M$ of a binary black hole prior to merging, and the portion $\xi$ of the 
mass of black holes emitted in the form of gravitational waves.

To study the impact heating and adiabatic expansion effects in geometrically thin discs, we may leave apart a detailed description of the vertical structure. Instead, we may use a simple approximation that, at any time moment, the vertical velocity $v_{z}$ is proportional to the height $z$ (the analogue of the Hubble law in cosmology):

$$
V_{z}(r, z, t)=\psi(r, t) z .
$$

In the plane of symmetry of a disc, the vertical component of the velocity $v_{z}=0$. In our model, we write all Eqs. (6) -(10) for the plane of symmetry of a disc. In this case, the terms like $v_{z} \partial / \partial z$ in convective derivatives disappear in all of the equations. Considering this note and approximation (11), Eqs. (6)-(10) (except (8)) take the form:

$$
\begin{gathered}
\frac{\partial \rho}{\partial t}+v_{r} \frac{\partial \rho}{\partial r}+\rho \frac{1}{r} \frac{\partial}{\partial r}\left(r v_{r}\right)+\rho \psi=0 \\
\frac{\partial v_{r}}{\partial t}+v_{r} \frac{\partial v_{r}}{\partial r}-\frac{v_{\varphi}^{2}}{r}+\frac{1}{\rho} \frac{\partial P}{\partial r}+(1-\xi) \frac{G M}{r^{2}}=0 \\
\frac{\partial v_{\varphi}}{\partial t}+v_{r} \frac{\partial v_{\varphi}}{\partial r}+\frac{v_{r} V_{\varphi}}{r}=0 \\
\frac{\partial \varepsilon}{\partial t}+v_{r} \frac{\partial \varepsilon}{\partial r}+\frac{P}{\rho} \frac{1}{r} \frac{\partial}{\partial r}\left(r v_{r}\right)+\frac{P \psi}{\rho}=0
\end{gathered}
$$

The last term in the energy equation (Eq. (15)) describes the adiabatic heating of the disc caused by its vertical motions.

Let us assume that, at an arbitrary time moment, the pressure in the disc is determined by the expression

$$
P(r, z, t)=P(r, t) e^{-z^{2} / H^{2}(r, t)},
$$

where $H$ is the local half-thickness of the disc. Then, from the equation for the vertical velocity (Eq. (8)) and with using relationship (11), we may derive the equation for the coefficient $\psi$ :

$$
\frac{\partial \psi}{\partial t}+v_{r} \frac{\partial \psi}{\partial r}+\psi^{2}-\frac{2 P}{\rho H^{2}}+(1-\xi) \frac{G M}{r^{3}}=0 .
$$

By introducing $z=H(r, t)$ into relationship (11), we find

$$
V_{z}(r, H(r, t), t)=\psi(r, t) H(r, t) .
$$

On the other side, we may write

$$
v_{z}(r, H(r, t), t)=\frac{\partial H}{\partial t}+v_{r} \frac{\partial H}{\partial r} .
$$

Thus, the local half-thickness of the disc $H(r, t)$ satisfies the equation

$$
\frac{\partial H}{\partial t}+v_{r} \frac{\partial H}{\partial r}-\psi H=0 .
$$

To close the obtained system of equations (12)(15), (17), and (20), we should consider the equations of state that determine the dependences of the pressure $P$ and the internal energy $\varepsilon$ on the density $\rho$ and the temperature $T$. Since we consider here only zone B of the accretion disc, we ignore the radiation pressure. However, it is worth noting that, in strongly perturbed regions of this zone, where high-intensity shock waves arise, the temperature may grow to so high values that the radiation pressure effects may be of considerable importance [12]. The equations of state may be written in the following form:

$$
\begin{gathered}
P=A_{\mathrm{gas}} \rho T, \\
\varepsilon=\frac{A_{\mathrm{gas}} T}{\gamma-1},
\end{gathered}
$$

where $A_{\text {gas }}=2 k_{\mathrm{B}} / m_{\mathrm{p}}$ is the gas constant, $k_{\mathrm{B}}$ is the Boltzmann constant, $m_{\mathrm{p}}$ is the proton mass, and $\gamma$ is the adiabatic index. For the completely ionized hydrogen plasma, the medium molecular weight should be assumed at $1 / 2$, while the adiabatic index $\gamma=5 / 3$.

As initial conditions for the density and the temperature, expressions (4) and (5) are used. The initial distributions for the density and the internal energy can be derived from equations of state (21) and (22):

$$
\begin{gathered}
P_{0}(r)=\rho_{*} c_{*}^{2}\left(\frac{r}{r_{*}}\right)^{-k_{\mathrm{d}}-k_{\mathrm{t}}}, \\
\varepsilon_{0}=\frac{c_{*}^{2}}{\gamma-1}\left(\frac{r}{r_{*}}\right)^{-k_{\mathrm{t}}},
\end{gathered}
$$

where the squared velocity of light is defined as $c_{*}^{2}=A_{\mathrm{gas}} T_{*}$. Moreover, we assume that, at the initial moment, $v_{r}(r, 0)=0$ and $\psi(r, 0)=0$. The initial conditions for the azimuthal velocity and the half-thickness of the disc can be obtained from the stationary solutions of Eqs. (13) and (17), which correspond to the unperturbed disc, when the parameter $\xi=0$ :

$$
\begin{gathered}
V_{\varphi}(r, 0)=c_{*}\left[\mu^{2}-\left(k_{\mathrm{d}}+k_{\mathrm{t}}\right)\left(\frac{r}{r_{*}}\right)^{1-k_{\mathrm{t}}}\right]^{1 / 2}\left(\frac{r}{r_{*}}\right)^{-1 / 2}, \\
H_{0}(r)=r_{*} \frac{\sqrt{2}}{\mu}\left(\frac{r}{r_{*}}\right)^{\frac{3-k_{\mathrm{t}}}{2}} .
\end{gathered}
$$

The dimensionless parameter

$$
\mu=\sqrt{\frac{G M}{r_{*} c_{*}^{2}}}
$$

determines the Mach number at the radius $r_{*}$, where $t_{*}=r_{*} / c_{*}$ is the characteristic time scale.

To calculate numerically the above equations, we use the implicit completely conservative difference 
scheme by Samarskii and Popov [38], some details of which are described in [39] (see Appendix A). It is worth stressing that, in this scheme, not only the difference analogues of the mass, momentum, and energy conservation laws but also the additional relationships, describing the balance on certain energy kinds, are fulfilled. Moreover, for our case, the scheme allows one to follow the difference analogue of the conservation law for the specific angular momentum. The numerical model is described by the following parameters: the indexes $k_{\mathrm{d}}$ and $k_{\mathrm{t}}$ for the initial profiles of the density and the temperature, respectively; the lost mass portion $\xi$; and the Mach number $\mu$. In a special case of $\psi=0$, it is important to note that this numerical model changes into a simpler model, which was considered in our paper [24] and where the effects due to adiabatic expansion in the disc are ignored.

We will assume that the dimensionless mass $m=55$ corresponds to the gravitational-wave event GW170814 (see Table 1). Let us choose the inner radius of an unperturbed disc $r_{\mathrm{d}}=6 R_{\mathrm{g}}$ to be a characteristic radius $r_{*}$, which determines the spatial scale of the problem. Then, when considering the temperature distribution in the unperturbed disc (5), we derive from (27) that

$$
\mu=78.02 \alpha^{1 / 10} \dot{m}^{-1 / 5} .
$$

If a value of the parameter $\dot{m}$ is fixed in this equation, we may obtain arbitrarily small values of the parameter $\mu$ in the limit at $\alpha \rightarrow 0$. On the contrary, if we fix a value of the parameter $\alpha$, we may obtain arbitrarily large values of the parameter $\mu$ in the limit at $\dot{m} \rightarrow 0$. For example, for $\alpha=0.01$ and $\dot{m}=1$, we obtain $\mu=$ 49.23. For $\alpha=0.01$ and $\dot{m}=0.01$, we obtain $\mu=$ 123.65. Hence, the possible values of the parameter $\mu$ are within rather wide limits.

Since we consider only zone B of an accretion disc, the characteristic radius $r_{*}$ can also be defined as an inner radius of this zone $r_{\mathrm{AB}}$. The value of this radius can be obtained from the condition of the same values for the radiation and gas pressure [33]

$$
r_{\mathrm{AB}}=159.3 \alpha^{2 / 21} m^{2 / 21} \dot{m}^{16 / 21} R_{\mathrm{g}} .
$$

From this expression and Eqs. (5) and (27), we derive

$$
\mu=64.97 \alpha^{2 / 21} \dot{m}^{-5 / 21} \text {. }
$$

This dependence of $\mu$ on $\alpha$ and $\dot{m}$ exhibits qualitatively the same behavior as that of dependence (28). The parameter $\mu$ becomes arbitrarily small in the limit at $\alpha \rightarrow 0$ and arbitrarily large in the limit at $\dot{m} \rightarrow 0$. Specifically, when $\alpha=0.01$ and $\dot{m}=1$, we obtain $\mu=41.9$; while for $\alpha=0.01$ and $\dot{m}=0.01, \mu=194.49$. These values are very close to those obtained for the previous case. Based on these estimates, in the calculations presented below, we varied the values of the dimensionless parameter $\mu$ from 50 to 200 .
As our calculations show [12, 13, 24], the perturbation degree of an accretion disc due to merging the black holes and losing the mass at the expense of radiating gravitational waves is determined by a value of the parameter $\mu$. It has turned out that the larger is the value of $\mu$, the higher is the electromagnetic response from the gravitational-wave event. In this regard, it is worth noting that, for supermassive black holes, the values of the parameter $\mu$ may be much larger than the estimates specified above for black holes of stellar masses. Indeed, let us choose the inner radius of an unperturbed disc to be the characteristic radius $r_{*}$. Then, by specifying the dimensionless mass as, for example, $m=10^{8}$, we derive

$$
\mu=329.73 \alpha^{1 / 10} \dot{m}^{-1 / 5} .
$$

Thus, it follows that $\mu=208.05$ for $\alpha=0.01$ and $\dot{m}=1$, while $\alpha=0.01$ and $\dot{m}=0.01$ yield $\mu=522.59$. For more massive black holes, even larger values of the parameter $\mu$ can be obtained.

\section{SELF-SIMILAR SOLUTION}

\subsection{Self-Similar Equations}

The analysis of the numerical solutions, which we obtained in a paper [24], allowed us to conclude that shock waves appearing in a perturbed disc are selfsimilar in the nature. In particular, the distance covered by the shock-wave front for the time $t$ is described by a power law of $t^{2 / 3}$, while its velocity decays with time as $t^{-1 / 3}$. In a paper [12], we analyzed whether the corresponding self-similar solution can be built. However, we failed to obtain it comprehensively because of the difficulties in determining analytically the position of shock waves. A complete self-similar solution was obtained in our paper [13] from the comparison of the self-similar and numerical solutions.

The self-similar solution describes the evolution of a perturbed disc, where shock waves propagate rather far away from its inner boundary. This region can be considered as corresponding to zone B of an accretion disk, and the radiation pressure does not substantially influence the dynamics of the matter behind the front of shock waves. In a paper [13], when building a selfsimilar solution, we also ignored the effects connected to the adiabatic expansion of a disc. It is exactly this simplified model that we considered in our paper [24]. In the present paper, we consider the self-similar solution that considers the adiabatic cooling of a disc.

We note that, in our model, the initial distributions of all of the quantities except the azimuthal velocity $v_{\varphi}$ and, consequently, the angular momentum $l=r v_{\varphi}$ are described by power-law functions of the radius $r$. However, it is easy to see that, for $k_{\mathrm{t}}=1$ (which is close 
to $9 / 10$ in the model [33] for zone B), the distribution also obeys a power law:

$$
l_{0}(r)=r_{*} c_{*}\left(\mu^{2}-k_{\mathrm{d}}-1\right)^{1 / 2}\left(\frac{r}{r_{*}}\right)^{1 / 2} .
$$

Equation (32) is a real-valued expression if $\mu^{2}>k_{\mathrm{d}}+1$. For example, $k_{\mathrm{d}}=33 / 20$ yields $\mu>1.63$.

Let us seek the solution in the following form [40]

$$
\begin{gathered}
\rho(r, t)=\rho_{0}(r) \sigma(\lambda), \\
v_{r}(r, t)=\frac{r}{t} u(\lambda), \\
l(r, t)=l_{0}(r) \Lambda(\lambda), \\
\varepsilon(r, t)=\varepsilon_{0}(r) w(\lambda), \\
\psi(r, t)=\frac{1}{t} \Psi(\lambda), \\
H(r, t)=H_{0}(r) h(\lambda),
\end{gathered}
$$

where the functions $\rho_{0}(r), l_{0}(r), \varepsilon_{0}(r)$, and $H_{0}(r)$ are determined (for $k_{\mathrm{t}}=1$ ) by expressions (4), (32), (24), and (26), respectively. The self-similar functions $\sigma, u$, $\Lambda, \Psi, w$, and $h$ depend on the self-similar variable $\lambda$, which is chosen as

$$
\lambda=\frac{r}{r_{*}}\left(\frac{t_{*}}{t}\right)^{\delta},
$$

where $\delta$ is the self-similarity index. This choice of the self-similar variable means that the limit at $\lambda \rightarrow \infty$ corresponds to the unperturbed state of the disc. Consequently, in the self-similar solution in the limit at $\lambda \rightarrow \infty$, the functions are $\sigma \rightarrow 1, u \rightarrow 0, \Lambda \rightarrow 1$, $w \rightarrow 1, \Psi \rightarrow 0$, and $h \rightarrow 1$. The parameter $\delta$ should be determined from the self-similarity condition of the initial equations.

With the self-similar variables, Eqs. (12)-(15), (17), and (20) can be written as follows:

$$
\begin{gathered}
(u-\delta) \frac{d \ln \sigma}{d \ln \lambda}+\frac{d u}{d \ln \lambda}+\left(2-k_{\mathrm{d}}\right) u+\Psi=0, \\
(u-\delta) \frac{d u}{d \ln \lambda}+u(u-1) \\
+\frac{w}{\lambda^{3}}\left(\frac{d \ln \sigma}{d \ln \lambda}+\frac{d \ln w}{d \ln \lambda}-k_{\mathrm{d}}-1\right) \\
-\left(\mu^{2}-k_{\mathrm{d}}-1\right) \frac{\Lambda^{2}}{\lambda^{3}}+(1-\xi) \frac{\mu^{2}}{\lambda^{3}}=0 \\
(u-\delta) \frac{d \ln \Lambda}{d \ln \lambda}+\frac{u}{2}=0 \\
(u-\delta) \frac{d \ln w}{d \ln \lambda}+(\gamma-1) \frac{d u}{d \ln \lambda} \\
+(2 \gamma-3) u+(\gamma-1) \Psi=0
\end{gathered}
$$

$$
\begin{gathered}
(u-\delta) \frac{d \Psi}{d \ln \lambda}+\Psi(\Psi-1)-\frac{w}{h^{2}} \frac{\mu^{2}}{\lambda^{3}}+(1-\xi) \frac{\mu^{2}}{\lambda^{3}}=0, \\
(u-\delta) \frac{d \ln h}{d \ln \lambda}+u-\Psi=0 .
\end{gathered}
$$

In this case, all of the dimensional coefficients are cancelled if $\delta=2 / 3$.

\subsection{Self-Similar Shock Waves}

In the self-similar solution, a constant value of the self-similar variable $\lambda_{\mathrm{s}}=$ const corresponds to the shock wave front. Hence, the position of a shock wave is

$$
r_{\mathrm{s}}=\lambda_{\mathrm{s}} r_{*}\left(\frac{t}{t_{*}}\right)^{\delta} \propto t^{\delta},
$$

while its velocity is

$$
D=\frac{d r_{\mathrm{s}}}{d t}=\delta \frac{r_{\mathrm{s}}}{t} \propto t^{\delta-1} .
$$

In a shock wave, the quantities should obey the Hugoniot conditions, which connect their values before and after the discontinuity (will be subscripted by 1 and 2, respectively). Our simple analysis [13] leads to the following relationships between the self-similar functions:

$$
\begin{gathered}
\sigma_{2} V_{2}=\sigma_{1} V_{1}, \\
\Lambda_{2}=\Lambda_{1}, \\
V_{2}=\frac{\gamma-1}{\gamma+1} \frac{1}{V_{1}}\left(V_{1}^{2}+\frac{2}{\gamma-1} W_{1}\right), \\
W_{2}=\left(\frac{\gamma-1}{\gamma+1}\right)^{2} \frac{1}{V_{1}^{2}}\left(V_{1}^{2}+\frac{2}{\gamma-1} W_{1}\right)\left(\frac{2 \gamma}{\gamma-1} V_{1}^{2}-W_{1}\right),
\end{gathered}
$$

where the following designations of the auxiliary quantities are used to simplify the presentation

$$
V=u-\delta, \quad W=\frac{\gamma w}{\lambda^{3}}
$$

These relationships remain also valid, if vertical motions in the disc are considered. In this case, the self-similar functions $\Psi$ and $h$ remain to be continuous on shock waves

$$
\Psi_{2}=\Psi_{1}, \quad h_{2}=h_{1} .
$$

As particularly follows from the Hugoniot conditions, the mass flux density $j=\sigma V$ and the angular momentum $\Lambda$ remain continuous when transiting through the shock wave front. Since $j \neq 0$ on shock waves, the value of $u$ cannot be equal to the value of $\delta$ either to the left or to the right of the discontinuity, i.e., $u \neq \delta$. Moreover, all shock waves in the disc propagate from the center to the periphery. Consequently, $j<0$, which leads to $u<\delta$. 


\subsection{Algebraic Integrals}

From self-similar equations (40)-(45), we may derive two algebraic integrals that describe the conservation laws for the angular momentum and the entropy. First of all, from Eqs. (40) and (45), we derive

$$
(u-\delta)\left(\frac{d \ln \sigma}{d \ln \lambda}+\frac{d \ln h}{d \ln \lambda}\right)+\frac{d u}{d \ln \lambda}+\left(3-k_{\mathrm{d}}\right) u=0 .
$$

From this, with accounting for Eq. (42), we obtain

$$
\begin{gathered}
(u-\delta)\left[\frac{d \ln \sigma}{d \ln \lambda}+\frac{d \ln h}{d \ln \lambda}-2\left(3-k_{\mathrm{d}}\right) \frac{d \ln \Lambda}{d \ln \lambda}\right] \\
+\frac{d u}{d \ln \lambda}=0 .
\end{gathered}
$$

The solution of this equation can be written in a form of

$$
\frac{\sigma h|u-\delta|}{\Lambda^{2\left(3-k_{\mathrm{d}}\right)}}=\text { const. }
$$

Since the quantities $\sigma V, \Lambda$, and $h$ remain continuous on shock waves, the integration constant in the obtained algebraic integral takes the same values in the entire computational domain. To determine this value, we may use the values of the self-similar functions $\sigma=1, u=0, \Lambda=1$, and $h=1$ at a limit of $\lambda \rightarrow \infty$. Thus, this constant is equal to the self-similarity index $\delta$. Consequently, we may write

$$
\Lambda^{2\left(3-k_{\mathrm{d}}\right)}=\frac{\sigma h}{\delta}|u-\delta| .
$$

This algebraic integral expresses the angular momentum conservation law in the self-similar form. It allows the function $\Lambda$ to be expressed explicitly through the functions $\sigma, u$, and $h$.

To derive the second algebraic integral, we use Eqs. (43) and (45) and find

$$
\begin{aligned}
& (u-\delta)\left[\frac{d \ln w}{d \ln \lambda}+(\gamma-1) \frac{d \ln h}{d \ln \lambda}\right] \\
& +(\gamma-1) \frac{d u}{d \ln \lambda}+(3 \gamma-4) u=0 .
\end{aligned}
$$

From this, considering Eq. (54), we obtain

$$
\begin{aligned}
& (u-\delta)\left\{(3 \gamma-4) \frac{d \ln \sigma}{d \ln \lambda}+\left[k_{\mathrm{d}}(\gamma-1)-1\right] \frac{d \ln h}{d \ln \lambda}\right. \\
& \left.-\left(3-k_{\mathrm{d}}\right) \frac{d \ln w}{d \ln \lambda}\right\}+\left[k_{\mathrm{d}}(\gamma-1)-1\right] \frac{d u}{d \ln \lambda}=0 .
\end{aligned}
$$

The solution of this equation can be written in a form of

$$
\frac{\sigma^{3 \gamma-4} h^{k_{\mathrm{d}}(\gamma-1)-1}}{w^{3-k_{\mathrm{d}}}}|u-\delta|^{k_{\mathrm{d}}(\gamma-1)-1}=\text { const. }
$$

This algebraic integral expresses the entropy conservation law (the adiabaticity integral) in the selfsimilar form. However, as distinct from the angular momentum conservation law, the entropy conservation law is followed only in the smooth solution domains, since, as follows from the Hugoniot conditions (48)-(53), the entropy of gas is not conserved in transition through the shock wave front. Consequently, the integration constant in Eq. (60) will be different for each of the smoothness domains. In the exterior smoothness domain (ahead of the first shock wave), the integration constant can again be determined by the limiting values of the self-similar functions at $\lambda \rightarrow \infty$. It turns out to be equal to $\delta^{(\gamma-1) k_{\mathrm{d}}-1}$. Hence, we may write

$$
w^{3-k_{\mathrm{d}}} \delta^{k_{\mathrm{d}}(\gamma-1)-1}=\sigma^{3 \gamma-4}(h|u-\delta|)^{k_{\mathrm{d}}(\gamma-1)-1} .
$$

From this relationship, the function $w$ in an exterior domain of the perturbed disc can be expressed explicitly through the functions $\sigma, u$, and $h$.

In the analytical model for the perturbation induced in an accretion disc by merging binary black holes (see our recent paper [13]), the adiabatic heating-cooling effects caused by vertical motions were ignored. The present model takes them into account. To pass to the simpler model [13], one should specify $\Psi=0$. In this specific case, from Eqs. (42) and (45), we obtain $h=\Lambda^{2}$. The corresponding substitution in the algebraic integral of the angular momentum (57) results in

$$
\Lambda^{2\left(2-k_{\mathrm{d}}\right)}=\frac{\sigma}{\delta}|u-\delta| .
$$

By eliminating $\Lambda^{2}$ from the above equation, we can write

$$
h^{2-k_{\mathrm{d}}}=\frac{\sigma}{\delta}|u-\delta| .
$$

While considering this relationship, we obtain (for an exterior part of the perturbed disc) the following expression from the algebraic adiabaticity integral

$$
w^{2-k_{\mathrm{d}}} \delta^{k_{\mathrm{d}}(\gamma-1)-1}=\sigma^{2 \gamma-3}|u-\delta|^{k_{\mathrm{d}}(\gamma-1)-1} .
$$

It is exactly the form in which the algebraic integrals for the angular momentum and the adiabaticity were written in our paper [13].

\subsection{Asymptotic Forms of the Self-Similar Functions}

Let us examine an asymptotic form of the self-similar functions in the limit at $\lambda \rightarrow \infty$. This asymptotic form describes the short-term growth of perturbations in an accretion disc. On the other hand, it corresponds to the state of the disc at an arbitrary time moment and large distances $r \gg r_{*}$ from the central object. While considering the limiting values of the self-similar functions $\sigma=1, u=0, \Lambda=1, w=1, \Psi=0$, and $h=1$ at $\lambda \rightarrow \infty$, the desired solution can be presented in a form of $u=u_{1}, \sigma=1+\sigma_{1}, \Lambda=1+\Lambda_{1}, w=1+w_{1}$, $\Psi=\Psi_{1}$, and $h=1+h_{1}$, where $u_{1}, \sigma_{1}, \Lambda_{1}, w_{1}, \Psi_{1}$, and $h_{1}$ are small quantities.

Note that, in the equation of motion (Eq. (41)), the main term at a limit of large values of $\lambda$, which determines the desired asymptotics, is the last term on the 
left-hand side. When all of the quantities that are small relative to it are cast out from this equation, we come to the following equation

$$
\delta \lambda u_{1}^{\prime}+u_{1}+\frac{\xi \mu^{2}}{\lambda^{3}}=0
$$

where the derivative with respect to the self-similar variable $\lambda$ is primed. Thus, it follows that

$$
u_{1}=\frac{\xi \mu^{2}}{\lambda^{3}} .
$$

Equation (44) may analogously be transformed, which yields

$$
\delta \lambda \Psi_{1}^{\prime}+\Psi_{1}+\frac{\xi \mu^{2}}{\lambda^{3}}=0 .
$$

From solving this equation, we obtain

$$
\Psi_{1}=\frac{\xi \mu^{2}}{\lambda^{3}}
$$

The discontinuity equation (Eq. (40)) at the considered limit can be rewritten as follows

$$
\delta \lambda \sigma_{1}^{\prime}-\lambda u_{1}^{\prime}-\left(2-k_{\mathrm{d}}\right) u_{1}-\Psi_{1}=0 .
$$

By substituting Eqs. (66) and (68) into Eq. (69), we obtain

$$
\sigma_{1}=\frac{k_{\mathrm{d}}}{2} \frac{\xi \mu^{2}}{\lambda^{3}} .
$$

The equation for the angular momentum will be written as follows

$$
\delta \lambda \Lambda_{1}^{\prime}-\frac{1}{2} u_{1}=0 .
$$

By substituting Eq. (66) into this equation, we obtain

$$
\Lambda_{1}=-\frac{1}{4} \frac{\xi \mu^{2}}{\lambda^{3}}
$$

Finally, the equation for the energy can be rewritten as follows

$$
\delta \lambda w_{1}^{\prime}-(\gamma-1) \lambda u_{1}^{\prime}-(2 \gamma-3) u_{1}-(\gamma-1) \Psi_{1}=0 .
$$

By substituting Eqs. (66) and (68) into this equation, we obtain

$$
w_{1}=\frac{1}{2} \frac{\xi \mu^{2}}{\lambda^{3}} .
$$

If Eqs. (66) and (68) are substituted into Eq. (45) for $h$, the functions $u_{1}$ and $\Psi_{1}$ are canceled. This means that the infinitesimal order of the function $h_{1}$ is higher. Consequently, to find the asymptotics of this self-similar function, it is necessary to consider the further expansion terms. A more detailed analysis (see Appendix B) yields

$$
h_{1}=\frac{1}{24}\left[2 \mu^{2}-5\left(k_{\mathrm{d}}+1\right)\right] \frac{\xi \mu^{2}}{\lambda^{6}} .
$$

When passing to dimensional quantities, we obtain:

$$
\begin{gathered}
v_{r}=\xi G M \frac{t}{r^{2}}, \\
\psi=\xi G M \frac{t}{r^{3}}, \\
\frac{\rho-\rho_{0}}{\rho_{0}}=\frac{1}{2} k_{\mathrm{d}} \xi G M \frac{t^{2}}{r^{3}}, \\
\frac{l-l_{0}}{l_{0}}=-\frac{1}{4} \xi G M \frac{t^{2}}{r^{3}}, \\
\frac{\varepsilon-\varepsilon_{0}}{\varepsilon_{0}}=\frac{1}{2} \xi G M \frac{t^{2}}{r^{3}}, \\
\frac{H-H_{0}}{H_{0}}=\frac{1}{24}\left[2 \mu^{2}-5\left(k_{\mathrm{d}}+1\right)\right] \xi G M \frac{t^{4}}{r^{6}} .
\end{gathered}
$$

In other words, the perturbations in the velocity grow linearly (in proportion to $t$ ); the perturbations in the density, angular momentum, and internal energy grow according to the square law (in proportion to $t^{2}$ ); while the perturbations in the half-thickness of a disc grow proportionally to $t^{4}$. In addition, the growth rate of all of these perturbations is in direct proportion to the mass portion $\xi$ lost when black holes are merging.

An asymptotic form of the self-similar functions in the opposite limit at $\lambda \rightarrow 0$ describes the transition of a perturbed accretion disc to a new stationary state on large time scales $t \gg t_{*}$. Due to the self-similarity of the problem, the asymptotics also corresponds to the state of a disc near the central object at an arbitrary time moment. Let us assume that, in the limit at $\lambda \rightarrow 0$, the self-similar functions are $u \rightarrow 0, \sigma \rightarrow \sigma_{0}$, $w \rightarrow w_{0}, \Lambda \rightarrow \Lambda_{0}, \Psi \rightarrow 0$, and $h \rightarrow h_{0}$. Then, from Eq. (41), we may obtain

$$
\left(k_{\mathrm{d}}+1\right) w_{0}+\left(\mu^{2}-k_{\mathrm{d}}-1\right) \Lambda_{0}^{2}=(1-\xi) \mu^{2} .
$$

Equation (44) at the same limit leads to the equality

$$
w_{0}=(1-\xi) h_{0}^{2} .
$$

Finally, the algebraic integral of the angular momentum allows the following expression to be written

$$
\Lambda_{0}^{2\left(3-k_{\mathrm{d}}\right)}=\sigma_{0} h_{0} .
$$

This system of three equations is incomplete, since it contains four unknown quantities $\sigma_{0}, \Lambda_{0}, w_{0}$, and $h_{0}$. The fourth missing equation could be the algebraic adiabaticity integral (Eq. (60)). However, since the solution contains shock waves, the corresponding constant cannot analytically be determined. Thus, 

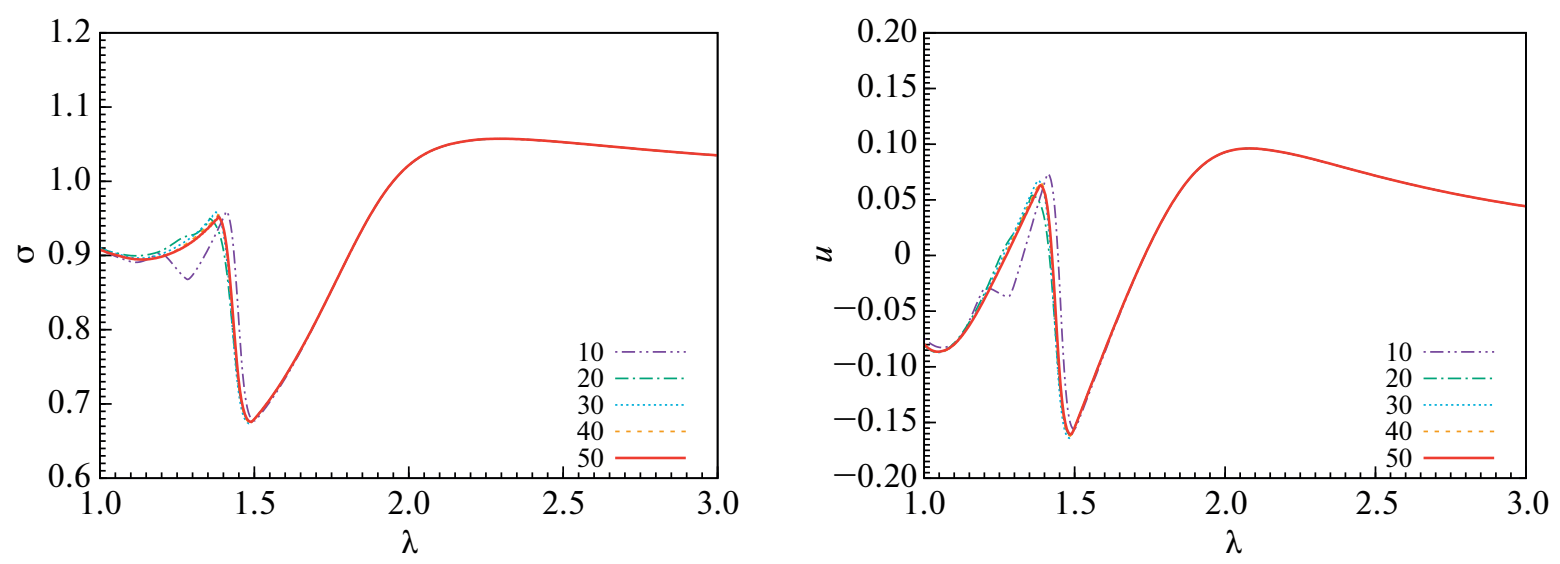

Fig. 1. The convergence of the numerical solution, describing the accretion disc perturbation due to merging the black holes and losing the mass, to a self-similar solution for the model with the parameter $\mu=5$. The numerical dependences $\sigma(\lambda)($ left $)$ and $u(\lambda)$ (right) are presented for different time moments $t / t_{*}$.

from these three equations, three unknown quantities can be expressed through the fourth unknown one. For example, the quantity $\sigma_{0}$ may be chosen to play this role. The value of the remained parameter $\sigma_{0}$ may be found from the numerical solution of self-similar equations, within which the positions of all shock waves are determined and the self-similar functions are joint by the Hugoniot conditions (48)-(53).

\section{CALCULATION RESULTS}

As previously shown in Section 3, for the problem under study, we have shown that a self-similar solution exists. Some of its properties have been also analyzed at length. The results of numerical calculations of the complete problem allow us to state that the solution really converges to a self-similar one with the course of time. The convergence of the numerical solution of Eqs. (33)-(36) to a self-similar one is illustrated in Fig. 1. This figure shows the numerical dependences $\sigma(\lambda)$ (left) and $u(\lambda)$ (right) for different time moments $t / t_{*}$. The solutions correspond to a value of the parameter $\mu=5$. As a result of merging the black holes and losing the mass, the accretion disc experiences perturbations; and a shock wave of weak intensity is formed within it. This wave propagates according to the selfsimilar law $\lambda=1.428$ with the self-similarity index $\delta=2 / 3$. In this case, in the domain ahead of a shock wave, the solution becomes self-similar almost straight away. Calculations show that, for large values of $\mu$, the self-similarity is attained much quicker. Hence, together with a numerical solution, it makes sense to consider a self-similarity solution as well.

We performed a series of numerical calculations of the accretion disc perturbation caused by merging black holes and losing the mass. While considering the estimates given at the end of Section 2, we specified the parameter $\mu$ in a range of $50 \leq \mu \leq 200$ (see Table 2).

In a paper [13], we considered a purely one-dimensional model of a perturbed accretion disc and built a self-similar solution by the following technique. First, for a rather large value of the self-similar variable $\lambda=$ $\lambda_{\max }$, we calculated the values of the self-similar functions according to the formulas that describe the asymptotics of the solution at a limit of $\lambda \rightarrow \infty$. These values were used as initial conditions. Then, by specifying a step of the self-similar function $\Delta \lambda<0$ and using the numerical method, we found the values of the quantities for $\lambda<\lambda_{\max }$. The number of shock waves and the positions of their fronts $\lambda_{s}$, where the subscript $s$ defines the number of a shock wave, cannot easily be found from the self-similar solution itself. We used this information on the shock waves from the corresponding numerical solution. As soon as the numerical integration of the self-similar equations yielded the value of the variable $\lambda$ equal to $\lambda_{1}$, the values of the functions at a point of $\lambda+\Delta \lambda$ were calculated with the Hugoniot conditions (48)-(53). Thus,

Table 2. Characteristics of the self-similar solutions

\begin{tabular}{r|c|c|c|c|c|c}
\hline$\mu$ & $\lambda_{1}$ & $\lambda_{2}$ & $\lambda_{3}$ & $\sigma_{0}$ & $w_{0}$ & $I_{\mu}$ \\
\hline 50 & 2.464 & 1.975 & 1.697 & 0.654 & 1.931 & 5.571 \\
100 & 3.779 & 3.028 & 2.585 & 0.403 & 5.069 & 297.066 \\
150 & 4.891 & 3.941 & 3.382 & 0.295 & 9.425 & 3741.66 \\
200 & 5.897 & 4.765 & 4.075 & 0.233 & 15.15 & 25304.2 \\
\hline
\end{tabular}

The columns contain the Mach number $\mu$; the self-similar coordinates of shock waves (in descending order) $\lambda_{1}, \lambda_{2}$, and $\lambda_{3}$; the values $\sigma_{0}$ and $w_{0}$ of the self-similar functions $\sigma(\lambda)$ and $w(\lambda)$ at a limit of $\lambda \rightarrow 0$; and the parameter $I_{\mu}$ that determines the bolometric luminosity of a perturbed disc according to Eq. (89). 

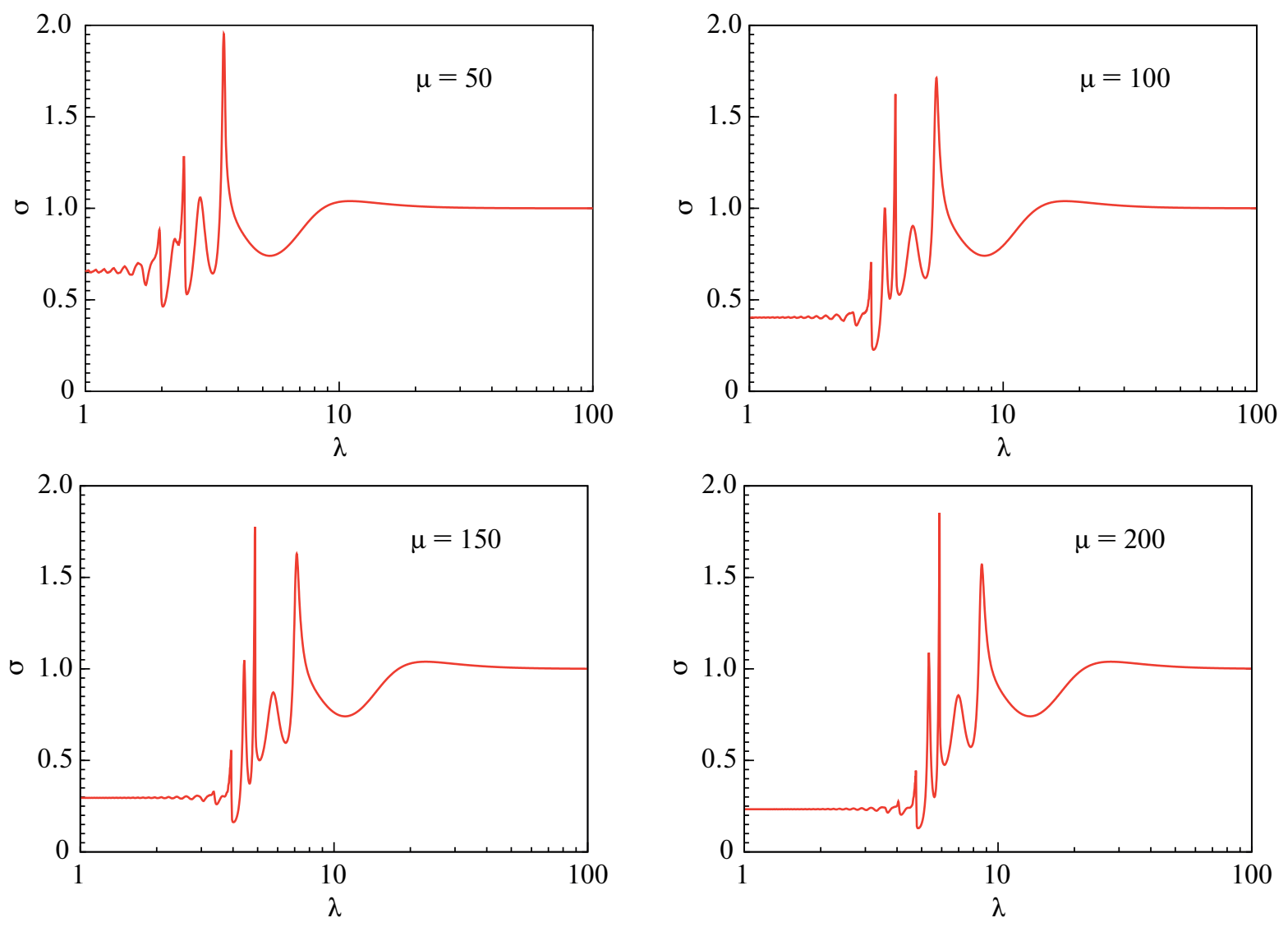

Fig. 2. The self-similar function $\sigma(\lambda)$ for the models with the parameter $\mu=50,100,150$, and 200 .

the integration procedure continued further to the next shock wave.

In the present paper, we consider the model corresponding to the 1.5-dimensional approximation, where vertical motions of matter in an accretion disc are considered. This results in a more complex behavior of the solution, and the above-described technique fails to provide a complete self-similar solution. The problem arises from the instability of the used RungeKutta scheme of the fourth order in the vicinity of critical points (see Appendix C), which are located immediately behind shock waves. To solve this problem correctly, it is necessary to use a better special-purpose numerical method. However, we did not do this, because we already had the self-similar solution, which was obtained from the numerical modeling of the complete problem. The self-similar solution, which was found by numerical calculations of the complete problem while considering the derived asymptotics, algebraic integrals of the angular momentum and adiabaticity, as well as the information about shock waves, allows one to estimate the magnitude and spectral characteristics of the electromagnetic response from the corresponding gravitational-wave event.
The solutions obtained for all of the models are presented in Figs. 2-7. These diagrams show that, according to all of the models, three shock waves are formed in an accretion disc and follow each other. The self-similar coordinates $\lambda_{s}$ of these shock waves are listed in Table 2. In the model with the maximal parameter $\mu=200$, one more shock wave, the fourth one, is actually formed, but its intensity is very weak; the corresponding self-similar coordinate is $\lambda_{s}=$ 3.622. The domain of strong perturbations, where shock waves are located, is in a relatively narrow interval of the self-similar variable $2<\lambda<10$. When the parameter $\mu$ grows, the perturbation energy is redistributed between the shock waves that unload this perturbation. The angular momentum $\Lambda(\lambda)$ (see Fig. 4) and the self-similar functions $\Psi(\lambda)$ (see Fig. 6) and $h(\lambda)$ (see Fig. 7) remain to be continuous on shock waves, as follows from the Hugoniot conditions (49) and (53) we obtained. The other self-similar functions undergo discontinuities.

From the analysis of the figures, we may conclude that the intensity of the first (outermost) shock wave gradually decreases, while the inner shock waves gradually grow in intensity. This is especially true of the second (intermediate) shock wave. As in the case for $\mu=200$, the perturbation energy is mainly concen- 

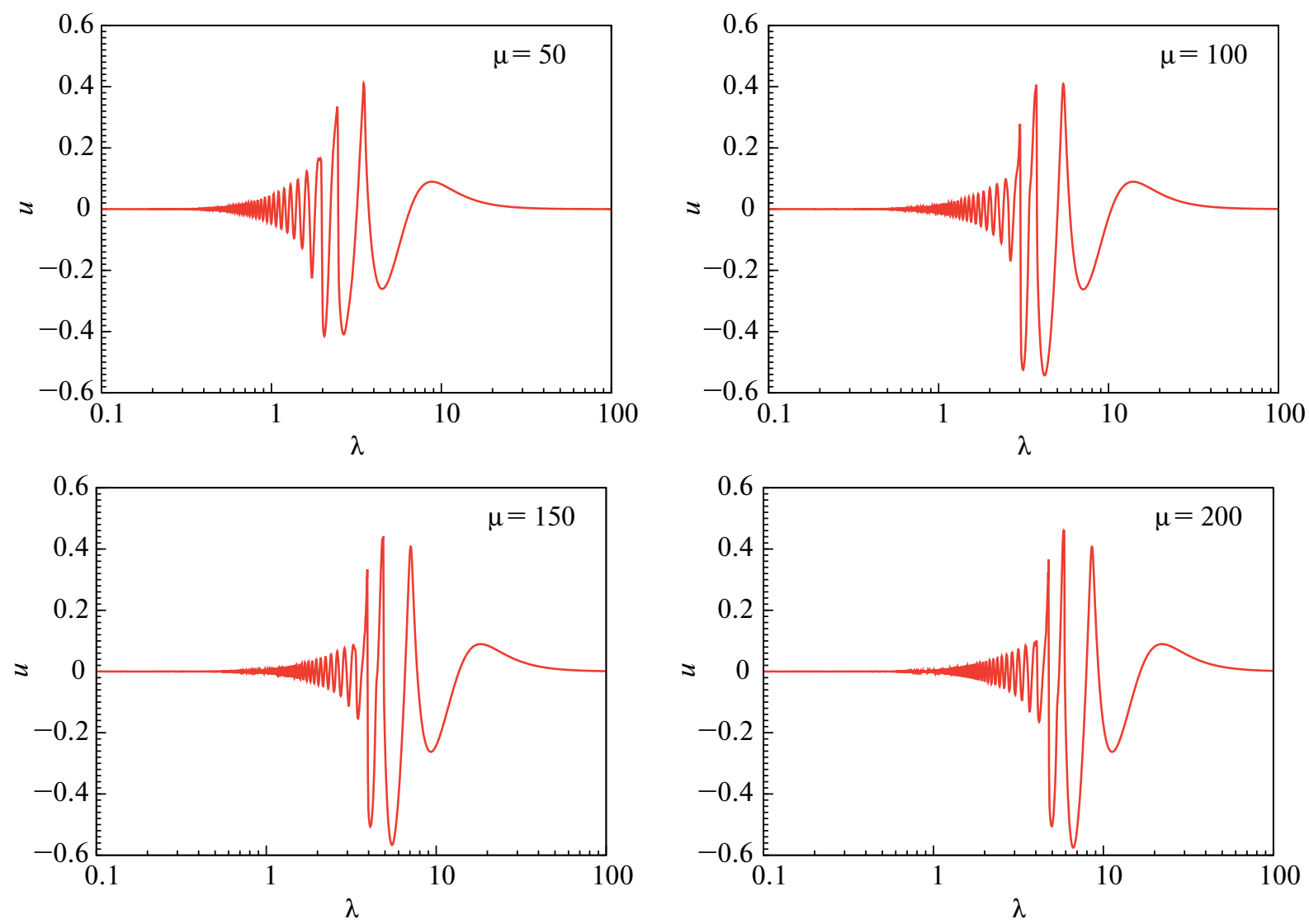

Fig. 3. The self-similar function $u(\lambda)$ for the models with the parameter $\mu=50,100,150$, and 200 .

trated in the second shock wave. It is interesting to note that each of the shock waves is preceded by nonlinear precursors propagating ahead of it. They are especially well seen in the distribution $w(\lambda)$ (see Fig. 5). In this case, immediately ahead of the outermost shock wave, there are even two precursors of this kind, while the precursor of the innermost shock wave is weak in intensity.

In the exterior domain ahead of shock waves, the self-similar solution is smooth; and, when $\lambda$ takes large values, it is described by the asymptotic expressions obtained in subsection 3.4. In the interior domain behind shock waves, when $\lambda$ takes small values, the self-similar solution also transforms into the corresponding asymptotics at $\lambda \rightarrow 0$. From verifying the calculation results, one can see that the algebraic integral of the angular momentum (57) is satisfied in a whole domain of the self-similar variable. At the same time, the algebraic integral of adiabaticity (61) strictly coincides with the solution obtained only for the exterior domain ahead of the first shock wave.

In the domain behind shock waves, the structure of an accretion disc becomes essentially nonstationary. The distribution of the radial velocity $u(\lambda)$ exhibits oscillations and reverses sign many times (see Fig. 3). This means that some parts of the disc move outward $\left(v_{r}>0\right)$, while the others, on the contrary, inward $\left(v_{r}<0\right)$. The vertical structure of the disc also experiences strong variations. The self-similar function $\Psi(\lambda)$, which describes the vertical velocity (see Eqs. (11) and (37)), quickly changes with time and exhibits well expressed oscillations (see Fig. 6). This results in quasi-periodic changes of the half-thickness of the disc $h(\lambda)$ (see Fig. 7), the amplitude of which gradually decays.

Once the nonlinear and shock waves have passed through the disc, a new stationary state, within which the values of the radial and vertical velocity are equal to zero, is formed there. This new state is characterized by a lower density, a higher temperature, and an enhanced thickness of the disc. The analysis of the obtained solution (see Table 2) allows us to conclude that, due to vertical expansion of the disc, the density in the symmetry plane drops by more than two times according to all of the models (except that for the parameter $\mu=50$, where the factor is 1.53 ), as compared to the unperturbed state.

\section{ELECTROMAGNETIC RESPONSE}

Due to propagation of shock waves through a disc, the matter is heated. A new stationary state of the disc, 

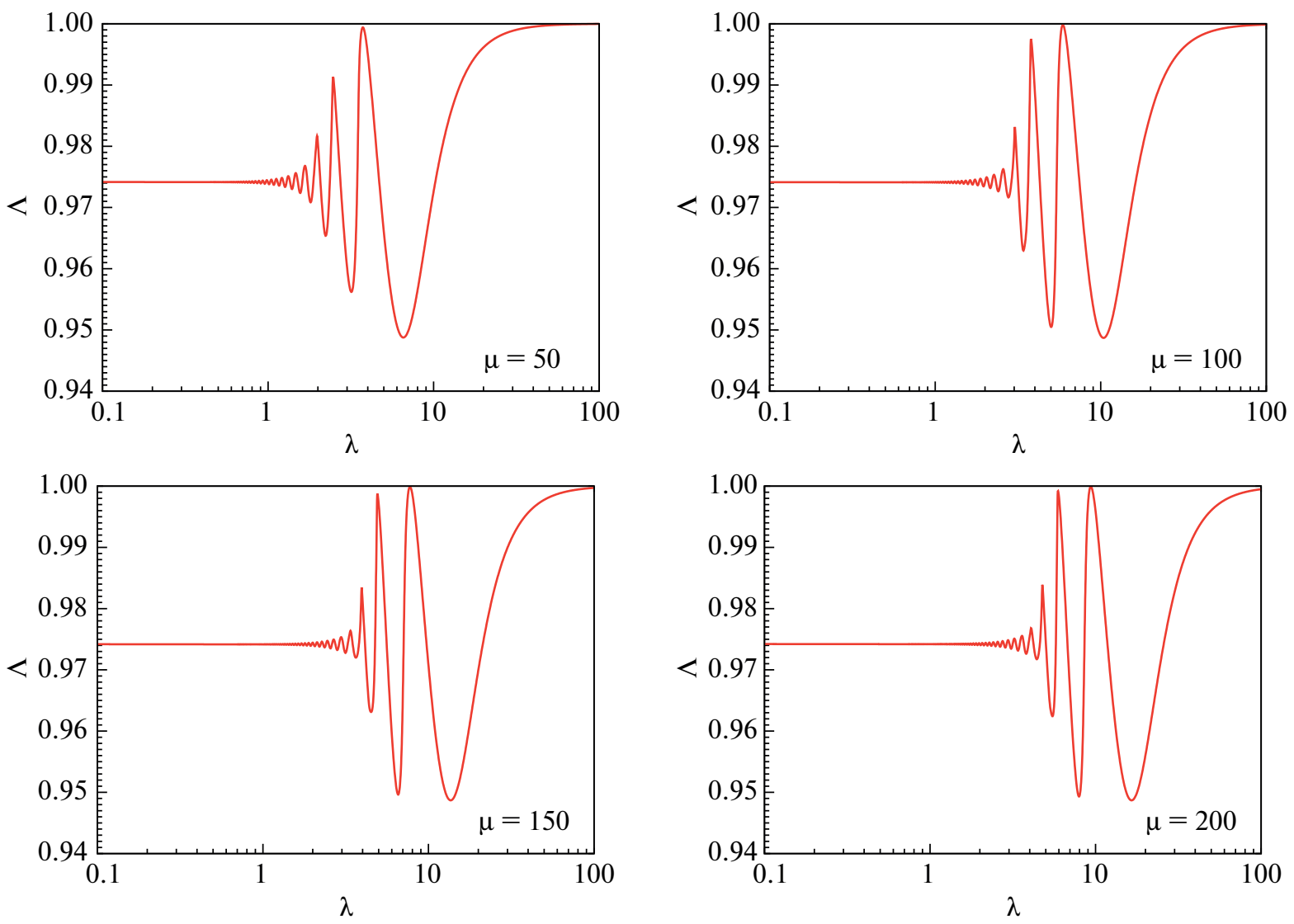

Fig. 4. The self-similar function $\Lambda(\lambda)$ for the models with the parameter $\mu=50,100,150$, and 200 .

which starts to be formed in its inner part behind the shock waves, is characterized by a higher temperature. As a result, the luminosity of the accretion disc sharply increases. This phenomenon may be considered as an electromagnetic response of the object to merging the black holes and losing the mass caused by the gravitational-wave emission.

The bolometric luminosity of an accretion disk (if viewed from one of the sides) can be calculated by the following expression

$$
L(t)=2 \pi^{2} \int_{r_{\mathrm{d}}}^{\infty} d r r \sigma_{\mathrm{SB}} T_{\mathrm{eff}}^{4}(r, t),
$$

where $\sigma_{\mathrm{SB}}$ is the Stefan-Boltzmann constant and $T_{\text {eff }}$ is the local effective temperature (at a specified radius $r$ ). The effective temperature $T_{\text {eff }}$ is not equal to the temperature $T$ in the symmetric plane, since the accretion disc in zone B is optically thick and, consequently, the radiation comes from the outer layer. In this case, we may assume that the emission of the disc in zone B exhibits the Planck spectrum. The temperature of matter in this outer layer is determined by the processes of the thermal energy transfer in a vertical direction from the symmetry plane of the disc to its surface.
To estimate the effective temperature in a geometrically thin disc, a simple approximation can be used; according to this approximation, $T_{\text {eff }}=f T$, where the coefficient $f$ is determined by a concrete process that transfers the thermal energy in a vertical direction [12, 24]. If the thermal energy is transferred by radiative heat conductivity, one may assume the coefficient $f=0.1$. If the vertical transfer of the thermal energy is determined by convection, the temperature difference may be estimated by a value of $f=0.5$.

The luminosity of a perturbed accretion disc can be estimated from the self-similar solution. The latter yields the following expression for the temperature in the plane of symmetry of the disc

$$
T(r, t)=T_{*} \frac{r_{*}}{r} w(\lambda) .
$$

Consequently, the bolometric luminosity (Eq. (85)) can be presented as

$$
L(t)=2 \pi \sigma_{\mathrm{SB}} f^{4} T_{*}^{4} r_{*}^{2}\left(\frac{t_{*}}{t}\right)^{4 / 3} \int_{\left(t_{*} / t\right)^{2 / 3}}^{\infty} \frac{w^{4}(\lambda) d \lambda}{\lambda^{3}},
$$



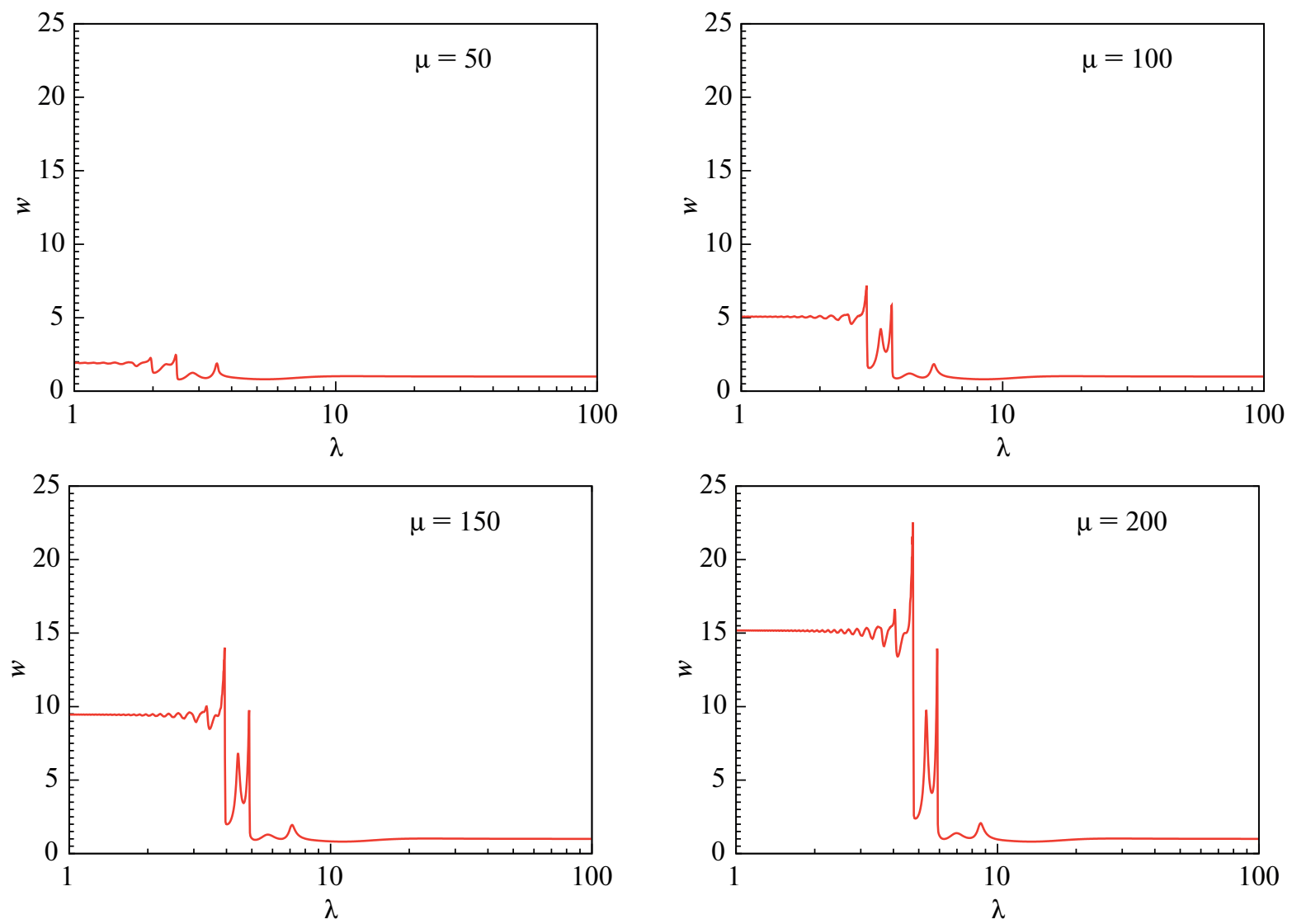

Fig. 5. The self-similar function $w(\lambda)$ for the models with the parameter $\mu=50,100,150$, and 200 .

where we assume $r_{\mathrm{d}}=r_{*}$. The integral with respect to the self-similar variable $\lambda$ can be written as a sum of two integrals over intervals of $\left(t_{*} / t\right)^{2 / 3} \leq \lambda \leq 1$ and $1 \leq \lambda \leq \infty$. In the first integral, the function $w(\lambda)$ can be substituted by its limit value $w_{0}$ with a proper accuracy at $\lambda \rightarrow 0$ :

$$
\int_{\left(t_{*} / t^{2 / 3}\right.}^{1} \frac{w^{4}(\lambda) d \lambda}{\lambda^{3}}=\frac{w_{0}^{4}}{2}\left[\left(\frac{t}{t_{*}}\right)^{4 / 3}-1\right] .
$$

The second integral

$$
I_{\mu}=\int_{1}^{\infty} \frac{w^{4}(\lambda) d \lambda}{\lambda^{3}}
$$

does not depend on time and can be calculated on the base of the obtained self-similar solution. Its concrete value is determined by the parameter $\mu$ (the last column in Table 2). As a result, we derive the following expression

$$
L(t)=2 \pi \sigma_{\mathrm{SB}} f^{4} T_{*}^{4} r_{*}^{2}\left(\frac{t_{*}}{t}\right)^{4 / 3}\left\{\frac{w_{0}^{4}}{2}\left[\left(\frac{t}{t_{*}}\right)^{4 / 3}-1\right]+I_{\mu}\right\}
$$

This function monotonically increases with time and, at a limit of $t \gg t_{*}$, approaches a constant value of

$$
L_{\max }=\pi w_{0}^{4} f^{4} \sigma_{\mathrm{SB}} T_{*}^{4} r_{*}^{2} .
$$

The dependences $L(t)$ obtained for the models with different values of the parameter $\mu$ and normalized to the limit value $L_{\max }$ are shown in Fig. 8. From the analysis of these light curves, we may conclude that, for the time of about a few characteristic time $t_{*}$ units (which is a few seconds), the luminosity of a perturbed disc reaches a constant value of $L_{\max }$. The growth in the luminosity is determined by a factor of $w_{0}^{4}$, the values of which for different models are listed in Table 2. When the parameter $\mu$ increases, the magnitude of the electromagnetic response substantially grows. For the model with $\mu=50$, the luminosity increases only by an order of magnitude; however, when $\mu=200$, the growth in the luminosity already exceeds four orders of magnitude.

Later, the heated matter will gradually get cold due to radiative cooling; and the luminosity of the perturbed disc will be slowly diminishing. We ignored this effect in the hydrodynamical model and the self-similar solution. However, the characteristic cooling time 

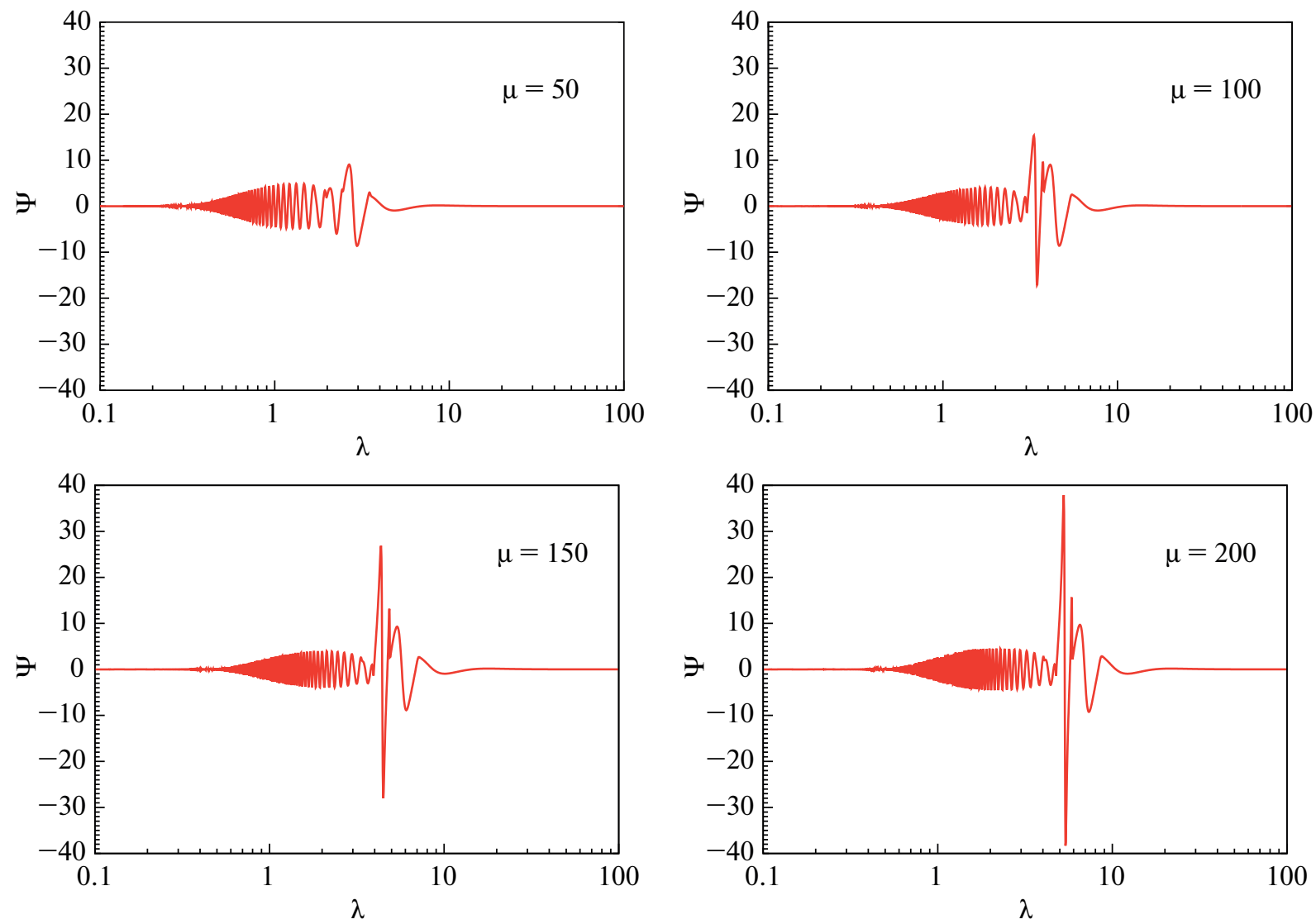

Fig. 6. The self-similar function $\Psi(\lambda)$ for the models with the parameter $\mu=50,100,150$, and 200 .

$t_{\text {cool }}$ can easily be estimated [13]. Under the diffuse approximation of the radiative transfer, the volume cooling coefficient may be written as

$$
\Lambda_{\text {cool }}=\frac{4 \sigma_{\mathrm{SB}} T^{4}}{3 \kappa \rho H^{2}}
$$

where $\kappa$ is the opacity coefficient, which is determined by the Thomson scattering in zone B. Hence, the characteristic cooling time is

$$
t_{\text {cool }}=\frac{\rho \varepsilon}{\Lambda_{\text {cool }}} .
$$

As follows from these results, at a point of $r=r_{*}$, the characteristic cooling time $t_{\text {cool }}$ is a few $t_{*}$ time units; consequently, this domain is cooling down rather rapidly. However, with increasing the distance, the cooling time $t_{\text {cool }}$ becomes larger. For example, at a distance of $r=20 r_{*}$, the cooling time is several hundred times higher than the time scale of the problem $t_{*}$.

The spectral density of the radiation flux from one side of the disc in the perpendicular direction,

$$
F_{\mathrm{v}}(t)=2 \pi^{2} \int_{r_{\mathrm{d}}}^{\infty} d r r B_{\mathrm{v}}\left(T_{\mathrm{eff}}(r, t)\right),
$$

is determined by the Planck function

$$
B_{v}(T)=\frac{2 h v^{3}}{c^{2}}\left(e^{\frac{h v}{k_{\mathrm{B}} T}}-1\right)^{-1},
$$

where $v$ is the frequency and $h$ is the Planck constant. To calculate the electromagnetic radiation spectrum, it is necessary to specify the parameters of an accretion disc and the quantities at its inner boundary. Because of this, we assumed $r_{\mathrm{d}}=r_{*}$ and, for certainty, specified the Shakura-Sunyaev parameter $\alpha=0.01$. Then, the temperature at the inner boundary of the disc $T_{*}$ may be calculated with formula (3), where the dimensionless accretion rate $\dot{m}$ is expressed through the parameter $\mu$ from relationship (28).

The obtained spectra of the electromagnetic radiation, which correspond to the self-similar solution, are presented in Fig. 9. The spectra based on different models for radiative discs $(f=0.1)$ and convective ones $(f=0.5)$ are shown in the left and right panels of Fig. 9, respectively. The bold and thin lines present the spectra of perturbed and unperturbed discs, respectively. The values of the fluxes $F_{v}$ are given in absolute units. The optical spectral range is indicated by a shaded bar. 

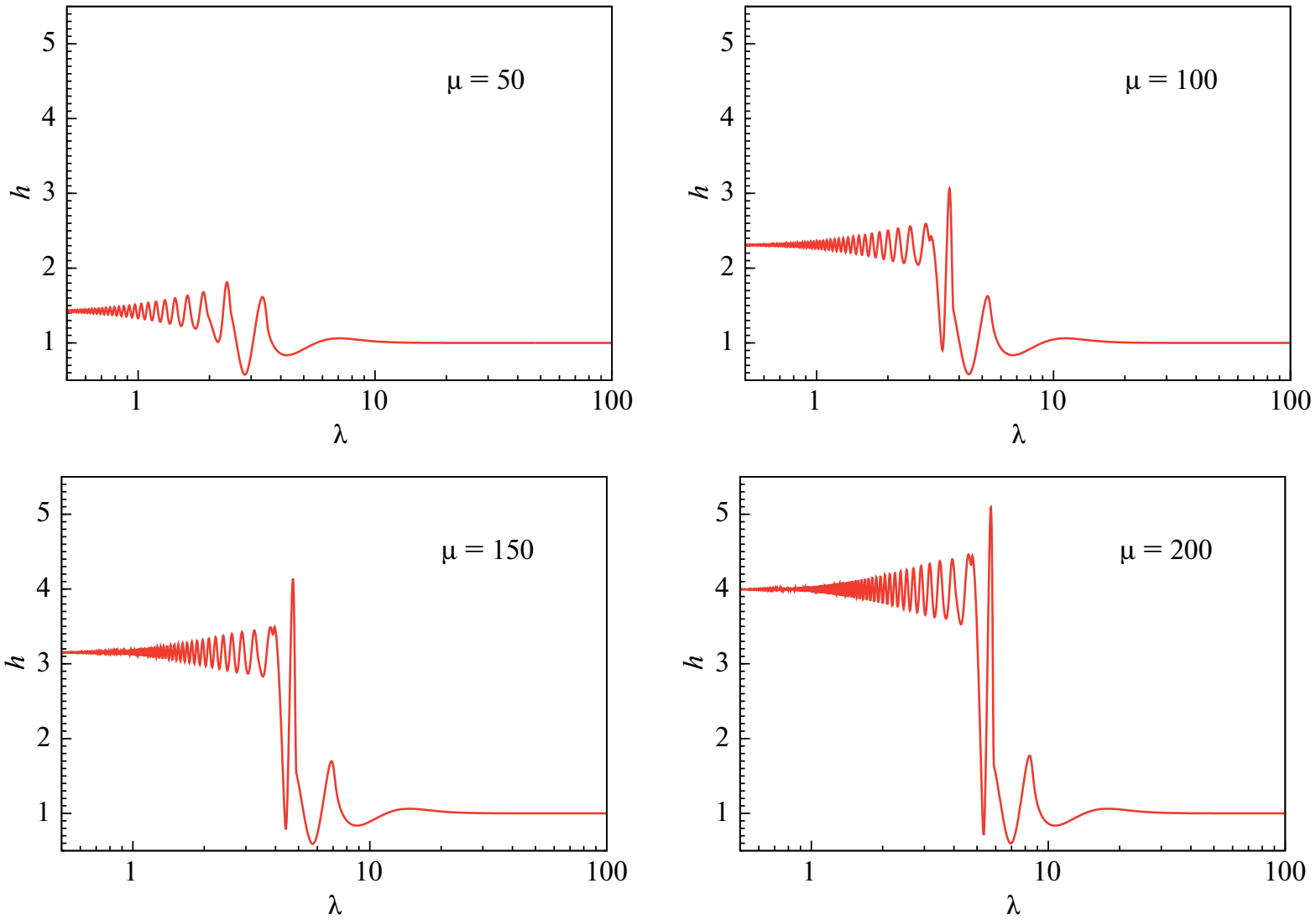

Fig. 7. The self-similar function $h(\lambda)$ for the models with the parameter $\mu=50,100,150$, and 200 .

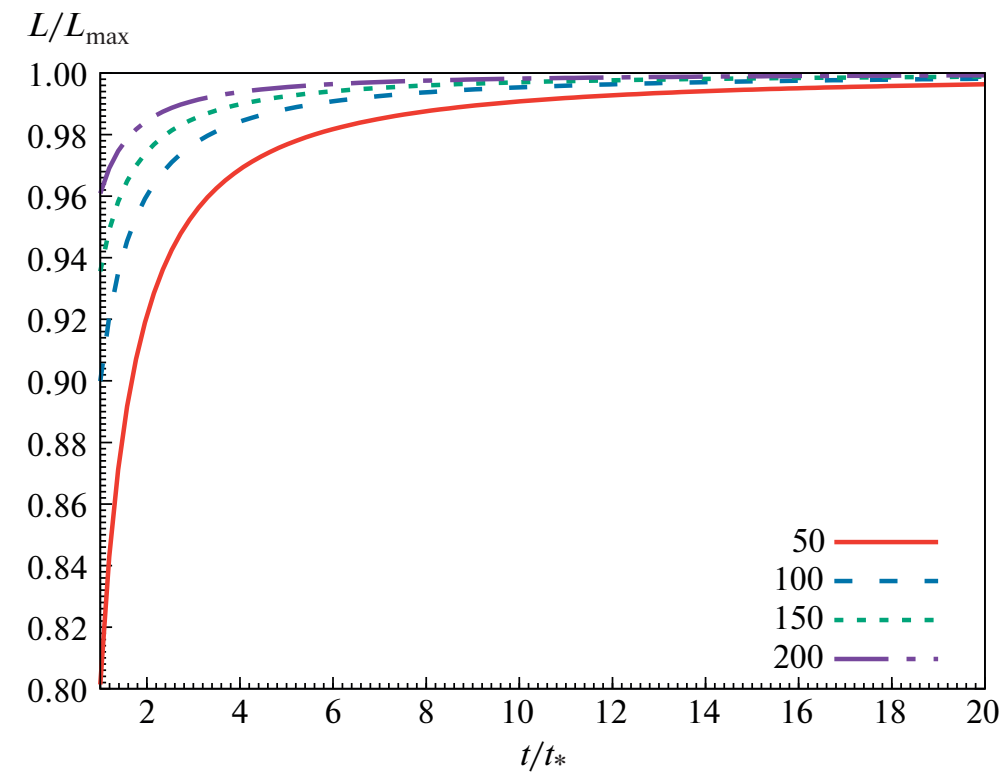

Fig. 8. The self-similar time dependence of the bolometric luminosity $L(t)$ for the models with the parameter $\mu=50,100,150$, and 200 . 

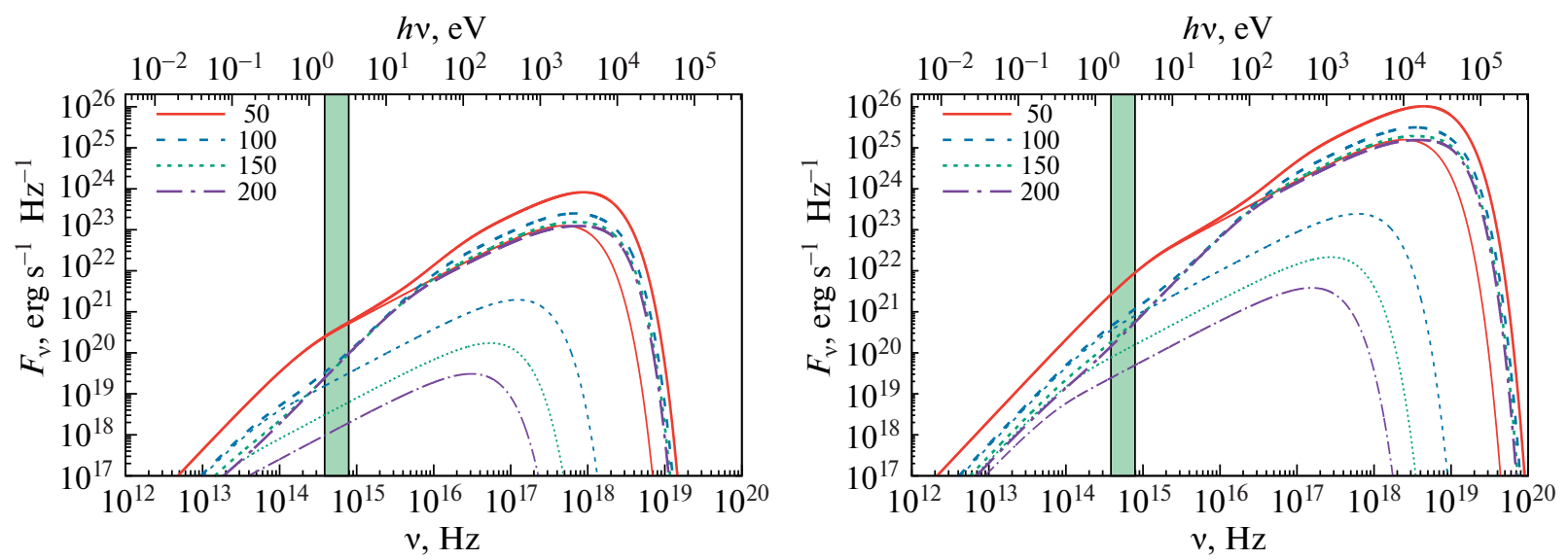

Fig. 9. The electromagnetic radiation spectra $F_{v}$ obtained from the self-similar solution for the radiative (left) and convective (right) discs. The bold and thin lines present the spectra of perturbed and unperturbed discs, respectively. The optical spectral range is indicated by a shaded bar.

As is seen from the diagrams, the radiative energy is mostly concentrated in a range of 1 to $100 \mathrm{keV}$, which corresponds to the soft and hard X-ray radiation. Moreover, in the gamma range $(\geq 100 \mathrm{keV})$, a portion of the coming radiative energy is also substantial. In the both cases (for radiative and convective discs), during an outburst, the maximal radiation flux generally grows by two orders of magnitude. At the same time, the frequency, at which the maximum is reached, moves toward high frequencies. In the case of convective discs, the radiation flux is two orders of magnitude higher than the flux from radiative discs. In addition, the maximal value of the flux from convective discs, as compared to that from radiative discs, is shifted to the hard X-ray range. In the visible spectral range, the radiation flux grows approximately by an order of magnitude, and the strongest relative effect of the outburst manifests itself when the parameter $\mu$ takes the largest value, $\mu=200$. Consequently, it should be expected that, when supermassive black holes are merging, the increase in the flux from the perturbed accretion disc should be much higher, which is also true for the optical spectral range.

\section{CONCLUSIONS}

We considered one of the probable cases of the electromagnetic response from the event of merging black holes. The scenario is based on the supposition that a binary black hole is surrounded by a circumbinary accretion disc. When black holes are merging, the mass of the target object decreases due to emission of gravitational waves. Consequently, the accretion disc receives the energy sufficient for perturbing it. Perturbations in the disc result in heating its matter, which induces a surge in the electromagnetic radiation flux from the disc. This model makes it possible to calculate the light curve and the spectral density of the radi- ation flux, as well as to estimate the characteristic duration of an outburst. The calculation results show that, for object GW170814, the luminosity burst from the accretion disc may be quite sufficient to provide detecting this response by state-of-art observational instruments.

In this paper, we examined the self-similar solution that describes the evolution of an accretion disc after merging black holes and losing the mass. In this case, the self-similarity index turned out to be $2 / 3$. The solution describes the structure of a perturbed disc in the domain (the so-called zone B), where the gas pressure dominates, while the opacity of matter is determined by the Thomson electron scattering processes. As distinct from our previous paper [13], here, we considered the effect caused by the adiabatic cooling of the disc. To build a self-similar solution, we used the numerical modeling results. From the self-similar equations, we derived the algebraic integrals of the angular momentum and the adiabaticity, which express the corresponding conservation laws. The algebraic integral of the angular momentum is satisfied in the entire range of the self-similar variable. The algebraic integral of the adiabaticity is satisfied only in the ranges of a smooth solution, since the entropy undergoes a discontinuity on shock waves. We also found the asymptotic relationships for the self-similar functions describing both the development of the initial perturbation and the transition of the disc to a new stationary state.

With the self-similar solution obtained for the problem, the magnitude of the electromagnetic response from the gravitational-wave event can approximately be estimated without time-consuming numerical calculations. The analysis of the self-similar solutions shows that, for the parameters corresponding to event GW170814, the luminosity of the disc may grow by one to four orders of magnitude. This may be 
quite sufficient to detect this response by state-of-art observational instruments.

It is worth stressing that the magnitude of the electromagnetic response substantially grows with increasing the Mach number at the inner boundary of the disc. We considered the merging of black holes with stellar masses, for which the characteristic value of this parameter is about 100. For supermassive black holes, this parameter may be significantly larger. Thus, it should be expected that, in the case of merging supermassive black holes, the electromagnetic radiation flux from a perturbed accretion disc will grow much higher, which is also true for the optical spectral range.

\section{APPENDIXA}

\section{A NUMERICAL METHOD}

To solve numerically Eqs. (12)-(15), (17), and (20), it is convenient to rewrite them in the dimensionless form. For this, the following quantities will be chosen as dimensional scales: $r_{0}=r_{*}, \quad v_{0}=c_{*}$, $t_{0}=r_{*} / c_{*}, \rho_{0}=\rho_{*}, P_{0}=\rho_{*} c_{*}^{2}, \varepsilon_{0}=c_{*}^{2}$, and $T_{0}=T_{*}$. To simplify the notation when describing the numerical model, we will designate the dimensionless quantities by the same letters as those for the corresponding dimensional ones.

To realize the numerical method of solving this system of equations, it is necessary to consider the Lagrangian mass variable. However, since the continuity equation (Eq. (12)) contains the additional term $\rho \Psi$, the mass of a column of liquid of a unit height is not conserved:

$$
\frac{d}{d t} \int_{0}^{r} \rho r d r \neq 0 .
$$

Consequently, this quantity cannot be used as the Lagrangian mass variable. To overcome this difficulty, together with the real liquid of the density $\rho$ that satisfies Eq. (12), we will consider some additional virtual liquid of the density $\tilde{\rho}$, which satisfies the following equation

$$
\frac{\partial \tilde{\rho}}{\partial t}+\frac{1}{r} \frac{\partial}{\partial r}\left(r \tilde{\rho} v_{r}\right)=0 .
$$

Evidently, this virtual liquid describes the same disc, but the latter is at a strict vertical hydrostatic equilibrium at any time moment.

In this case, we may introduce the Lagrangian mass variable as follows

$$
q=\int_{0}^{r} \tilde{\rho} r d r .
$$

Then, the following relationships turn out to be true:

$$
\frac{\partial}{\partial r}=r \tilde{\rho} \frac{\partial}{\partial q}, \quad \frac{1}{\tilde{\rho}}=r \frac{\partial r}{\partial q} .
$$

For the Lagrangian variables, Eq. (A.2) takes a form of

$$
\frac{d}{d t}\left(\frac{1}{\tilde{\rho}}\right)=\frac{\partial}{\partial q}\left(r v_{r}\right)
$$

where the total time derivative is determined as

$$
\frac{d}{d t}=\frac{\partial}{\partial t}+v_{r} \frac{\partial}{\partial r} .
$$

Let $\eta$ to stand for the ratio of the densities for the real and virtual liquids,

$$
\eta=\frac{\rho}{\tilde{\rho}} .
$$

Then, in (A.4), the second relationship can be rewritten as

$$
\eta=\rho r \frac{\partial r}{\partial q} .
$$

Hence, the system of Eqs. (12)-(15), (17), and (20) will take the following form:

$$
\begin{gathered}
\frac{d}{d t}\left(\frac{1}{\rho}\right)=\frac{1}{\eta} \frac{\partial}{\partial q}\left(r v_{r}\right)+\frac{\psi}{\rho}, \\
\frac{d v_{r}}{d t}=-\frac{r}{\eta} \frac{\partial P}{\partial q}+\frac{v_{\varphi}^{2}}{r}-(1-\xi) \frac{\mu^{2}}{r^{2}}, \\
\frac{d v_{\varphi}}{d t}=-\frac{V_{r} V_{\varphi}}{r}, \\
\frac{d \varepsilon}{d t}=-\frac{P}{\eta} \frac{\partial}{\partial q}\left(r V_{r}\right)-\frac{P}{\rho} \psi, \\
\frac{d \psi}{d t}=-\psi^{2}+\frac{P}{\rho H^{2}}-(1-\xi) \frac{\mu^{2}}{r^{3}}, \\
\frac{d H}{d t}=\psi H, \\
\frac{d r}{d t}=v_{r}, \\
P=\rho T, \\
\varepsilon=\frac{T}{\gamma-1} .
\end{gathered}
$$

Let us introduce a difference mesh into the computational domain in the coordinate system related to the Lagrangian mass coordinate $q$. The structure of this mesh is determined by the distribution for the coordinates of nodes $q_{i}$, where the index is $i=0,1, \ldots, N$. The mesh spacing $\Delta q_{i+1 / 2}=q_{i+1}-q_{i}$ was chosen in such a way that the logarithmic spaces between the neighbor nodes are the same, $\ln \left(q_{i+1} / q_{i}\right)=$ const. Let the radial coordinate $r$ and the velocities $V_{r}$ and $v_{\varphi}$ at the time moment $t^{n}$ to be related to the mesh nodes $r_{i}^{n}, v_{r, i}^{n}$, and $v_{\varphi, i}^{n}$, while the thermodynamic quantities $\rho, P, \varepsilon$, and $T$, as well as the quantities $\eta, \psi$, and $H$, to be related 
to the centers of the cells numbered by the half-integral indexes $\rho_{i+1 / 2}^{n}, P_{i+1 / 2}^{n}, \varepsilon_{i+1 / 2}^{n}, T_{i+1 / 2}^{n}, \eta_{i+1 / 2}^{n}, \psi_{i+1 / 2}^{n}$, and $H_{i+1 / 2}^{n}$ [38]. To make the further notations simpler, we will use the following designations for the finite difference operators

$$
\begin{aligned}
\left(\Delta_{t} f\right)^{n+1 / 2}= & \frac{f^{n+1}-f^{n}}{\Delta t}, \quad\left(\Delta_{q} f\right)_{i+1 / 2}=\frac{f_{i+1}-f_{i}}{\Delta q_{i+1 / 2}}, \\
& \left(\Delta_{q} f\right)_{i}=\frac{f_{i+1 / 2}-f_{i-1 / 2}}{\Delta q_{i}},
\end{aligned}
$$

where $\Delta q_{i}=\left(\Delta q_{i+1 / 2}+\Delta q_{i-1 / 2}\right) / 2$. The indexes in the operators may be omitted everywhere, if no confusion arises.

The above equations can be approximated by the following difference scheme:

$$
\begin{aligned}
& \Delta_{t} \frac{1}{\rho_{i+1 / 2}}=\frac{1}{\eta_{i+1 / 2}^{n+1 / 2}}\left[\Delta_{q}\left(r^{n+1 / 2} v_{r}^{n+1 / 2}\right)\right]_{i+1 / 2}+\frac{\psi_{i+1 / 2}^{n+1 / 2}}{\rho_{i+1 / 2}^{n+1 / 2}}, \\
& \Delta_{t} V_{r, i}=-\frac{r_{i}^{n+1 / 2}}{\eta_{i}^{n+1 / 2}}\left(\Delta_{q} \Pi^{n+\sigma}\right)_{i}+\frac{\left(V_{\varphi, i}^{n+1 / 2}\right)^{2}}{r_{i}^{n+1 / 2}} \\
& -(1-\xi) \frac{\mu^{2}}{r_{i}^{n+1} r_{i}^{n}}, \\
& \Delta_{t} V_{\varphi, i}=-\frac{V_{r, i}^{n+1 / 2} V_{\varphi, i}^{n+1 / 2}}{r_{i}^{n+1 / 2}}, \\
& \Delta_{t} \varepsilon_{i+1 / 2} \\
& =-\frac{\prod_{i+1 / 2}^{n+\sigma}}{\eta_{i+1 / 2}^{n+1 / 2}}\left[\Delta_{q}\left(r^{n+1 / 2} V_{r}^{n+1 / 2}\right)\right]_{i+1 / 2}-\frac{P_{i+1 / 2}^{n+1 / 2} \psi_{i+1 / 2}^{n+1 / 2}}{\rho_{i+1 / 2}^{n+1 / 2}}, \\
& \Delta_{t} r_{i}=v_{r, i}^{n+1 / 2} \\
& \Delta_{t} \psi_{i+1 / 2}=-\left(\psi_{i+1 / 2}^{n+1 / 2}\right)^{2} \\
& +\frac{P_{i+1 / 2}^{n+1 / 2}}{\rho_{i+1 / 2}^{n+1 / 2}\left(H_{i+1 / 2}^{n+1 / 2}\right)^{2}}-(1-\xi) \frac{\mu^{2}}{\left(r_{i+1 / 2}^{n+1 / 2}\right)^{3}}, \\
& \Delta_{t} H_{i+1 / 2}=\psi_{i+1 / 2}^{n+1 / 2} H_{i+1 / 2}^{n+1 / 2}, \\
& P_{i+1 / 2}^{n+1}=\rho_{i+1 / 2}^{n+1} T_{i+1 / 2}^{n+1}, \\
& \varepsilon_{i+1 / 2}^{n+1}=\frac{T_{i+1 / 2}^{n+1}}{\gamma-1}, \\
& \eta_{i+1 / 2}^{n+1}=\frac{1}{2} \rho_{i+1 / 2}^{n+1}\left[\Delta_{q}\left(r^{n+1}\right)^{2}\right]_{i+1 / 2}, \\
& \Pi_{i+1 / 2}^{n+1}=P_{i+1 / 2}^{n+1}+\omega_{i+1 / 2}^{n+1}, \\
& \omega_{i+1 / 2}^{n+1}=\Omega\left(\rho_{i+1 / 2}^{n+1}, r_{i}^{n+1}, r_{i+1}^{n+1}, v_{i}^{n+1}, v_{i+1}^{n+1}\right),
\end{aligned}
$$

where

$$
\Pi_{i+1 / 2}^{n+\sigma}=\sigma \Pi_{i+1 / 2}^{n+1}+(1-\sigma) \Pi_{i+1 / 2}^{n},
$$

$$
r_{i+1 / 2}=\frac{1}{2}\left(r_{i}+r_{i+1}\right) .
$$

The parameter $\sigma$ characterizes the implicitness degree of the scheme and varies from 0 to 1 . This parameter determines the approximation order relative to the time. For $\sigma=1 / 2$, the scheme has the second approximation order relative to $\Delta t$, while it is the first one in the other cases. The quantity $\omega$ describes the artificial viscosity, which is required to describe more correctly the solutions with shock waves and contact discontinuities. The explicit form of the function $\Omega$ is determined by a concrete model for the artificial viscosity. In our computations, we use the linear viscosity in the following form

$$
\omega=-\frac{v \rho}{2}\left(\frac{\partial v_{r}}{\partial r}-\left|\frac{\partial v_{r}}{\partial r}\right|\right),
$$

where the coefficient $v_{i+1 / 2}=v_{0} \Delta q_{i+1 / 2}$. The value $v_{0}$ was specified in an interval of 3 to 5 . The system of algebraic equations (A.19)-(A.30) is nonlinear. To solve it, the combination of the Newton method and the elimination one is used.

It can be shown that the difference scheme is completely conservative [38] in the sense that it provides the balance of specific energy kinds (thermal, kinetic, rotational, and gravitational) rather than only the total energy. Moreover, it is easy to show that, in this scheme, the difference relationship

$$
\Delta_{t}\left(r_{i} v_{\varphi, i}\right)=0
$$

which describes the conservation law of the angular momentum $l=r v_{\varphi}$, is obeyed. This means that the following equality takes place

$$
r_{i}^{n} V_{\varphi, i}^{n}=r_{i}^{0} V_{\varphi, i}^{0}=l_{i},
$$

where $l_{i}$ is the mesh function for the specific angular momentum. Consequently,

$$
v_{\varphi, i}^{n}=\frac{l_{i}}{r_{i}^{n}} .
$$

\section{APPENDIX B}

\section{AN ASYMPTOTIC FORM OF THE SELF-SIMILAR SOLUTION}

The asymptotic behavior of the self-similar functions at a limit of $\lambda \rightarrow \infty$ may be analyzed at length on the basis of their representation in the following form

$$
f=f_{\infty}+\frac{f_{3}}{\lambda^{3}}+\frac{f_{4}}{\lambda^{4}}+\frac{f_{5}}{\lambda^{5}}+\frac{f_{6}}{\lambda^{6}} .
$$

Here, $f=f(\lambda)$ is one of the self-similar functions $\sigma$, $u, \Lambda, w, \Psi$, and $h$. The free term $f_{\infty}$ is equal to zero for the functions $u$ and $\Psi$, while it is equal to unity for the other functions. The constants $f_{3}, f_{4}, f_{5}$, and $f_{6}$ are to be determined for each of the functions. 
By substituting these expressions to the self-similar equations (40)-(45), expanding them into a power series of a small parameter $1 / \lambda$, and gathering the terms at the same power indexes of this parameter, we come to the following. The values of the constants $f_{3}$ coincide with those we obtained from the direct asymptotic analysis of the initial equations:

$$
\begin{gathered}
\sigma_{3}=\frac{1}{2} k_{\mathrm{d}} \xi \mu^{2}, \quad u_{3}=\xi \mu^{2}, \quad \Lambda_{3}=-\frac{1}{4} \xi \mu^{2}, \\
w_{3}=\frac{1}{2} \xi \mu^{2}, \quad \Psi_{3}=\xi \mu^{2}, \quad h_{3}=0 .
\end{gathered}
$$

The constants $f_{4}=0$ and $f_{5}=0$. The values of the constants $f_{6}$ are determined by the expressions

$$
\begin{gathered}
\sigma_{6}=\frac{1}{24}\left[\left(k_{\mathrm{d}}+3\right)\left(3 k_{\mathrm{d}}+4\right) \xi \mu^{2}\right. \\
\left.-\left(k_{\mathrm{d}}+5\right) \mu^{2}+5\left(k_{\mathrm{d}}+1\right)\left(k_{\mathrm{d}}+4\right)\right] \xi \mu^{2}, \\
u_{6}=\frac{1}{6}\left[4 \xi \mu^{2}-\mu^{2}+5\left(k_{\mathrm{d}}+1\right)\right] \xi \mu^{2}, \\
\Lambda_{6}=-\frac{1}{96}\left[23 \xi \mu^{2}-2 \mu^{2}+10\left(k_{\mathrm{d}}+1\right)\right] \xi \mu^{2}, \\
w_{6}=\frac{1}{24}\left[4(3 \gamma+1) \xi \mu^{2}-(5 \gamma-4) \mu^{2}\right. \\
\left.+5(4 \gamma-3)\left(k_{\mathrm{d}}+1\right)\right] \xi \mu^{2}, \\
\psi_{6}=\frac{1}{6}\left(4 \xi \mu^{2}+\mu^{2}\right) \xi \mu^{2}, \\
h_{6}=\frac{1}{24}\left[2 \mu^{2}-5\left(k_{\mathrm{d}}+1\right)\right] \xi \mu^{2} .
\end{gathered}
$$

APPENDIXC

\section{CRITICAL POINTS}

In the system of the self-similar equations (40)(45), the equations for the angular momentum $\Lambda$ (42), the function $\Psi$ (44), and the function $h(45)$ is already solved relative to the corresponding derivatives. After simple transformations, the remained equations take the following form:

$$
\begin{aligned}
& \left(V^{2}-W\right) \frac{d \ln \sigma}{d \ln \lambda}=X, \\
& \left(V^{2}-W\right) \frac{d u}{d \ln \lambda}=Y, \\
& \left(V^{2}-W\right) \frac{d \ln w}{d \ln \lambda}=Z,
\end{aligned}
$$

where

$$
\begin{gathered}
X=Q-\left(2-k_{\mathrm{d}}\right) u V-\frac{k_{\mathrm{d}}(\gamma-1)-1}{\gamma} \frac{u W}{V}-\Psi V, \\
Y=\Psi W+\frac{2 \gamma-k_{\mathrm{d}}-1}{\gamma} u W-Q V,
\end{gathered}
$$

$$
\begin{gathered}
Z=(\gamma-1) Q-(2 \gamma-3) u V \\
+\frac{k_{\mathrm{d}}(\gamma-1)-1}{\gamma} \frac{u W}{V}-(\gamma-1) \Psi V, \\
Q=u(u-1)-\frac{k_{\mathrm{d}}+1}{\gamma} W \\
-\left(\mu^{2}-k_{\mathrm{d}}-1\right) \frac{\Lambda^{2}}{\lambda^{3}}+(1-\xi) \frac{\mu^{2}}{\lambda^{3}} .
\end{gathered}
$$

The system of ordinary differential equations (C.1) (C.3) contains a critical point (or even several critical points) $\lambda_{c}$, at which the coefficients $\left(V^{2}-W\right)$ at the derivatives vanish. If the derivatives at a critical point remain to be continuous, the right-hand sides of Eqs. (C.1)-(C.3) should also vanish. In this case, it is clear that all of them vanish simultaneously, because the following conditions are satisfied, if the relationship $V_{\mathrm{c}}^{2}=W_{\mathrm{c}}$ is accounted for,

$$
X_{\mathrm{c}} W_{\mathrm{c}}=-Y_{\mathrm{c}} V_{\mathrm{c}}, \quad Z_{\mathrm{c}} W_{\mathrm{c}}=-(\gamma-1) Y_{\mathrm{c}} V_{\mathrm{c}} .
$$

The constant value of the self-similar variable $\lambda=\lambda_{\mathrm{c}}$ is related to the position of the critical point in space

$$
r_{\mathrm{c}}=\lambda_{\mathrm{c}} r_{*}\left(\frac{t}{t_{*}}\right)^{\delta}
$$

and the velocity

$$
V_{\mathrm{c}}=\frac{d r_{\mathrm{c}}}{d t}=\delta \frac{r_{\mathrm{c}}}{t} .
$$

Consequently, the condition $V_{\mathrm{c}}^{2}=W_{\mathrm{c}}$, being expressed in the initial dimensional quantities, takes a form of

$$
\left(V_{r}-V_{\mathrm{c}}\right)^{2}=\frac{\gamma P}{\rho} .
$$

As follows from this relationship, the velocity of a critical point $v_{\mathrm{c}}$ relative to particles of gas is equal to the local velocity of sound. Since the velocity of a shock wave $D$ relative to particles of gas is supersonic, the critical point is located behind the shock wave front. If the solution contains several shock waves, there will be a critical point behind each of them.

\section{ACKNOWLEDGMENTS}

The authors are grateful to G.S. Bisnovatii-Kogan (the Space Research Institute of the Russian Academy of Sciences) for fruitful discussions.

\section{FUNDING}

This work was supported by the Russian Foundation for Basic Research, project no. 19-29-11013. 


\section{REFERENCES}

1. B. P. Abbott, R. Abbott, T. D. Abbott, M. R. Abernathy, et al., Astrophys. J. Lett. 818, L22 (2016).

2. L. D. Landau and E. M. Lifshits, Course of Theoretical Physics, Vol. 2: The Classical Theory of Fields (Fizmatlit, Moscow, 2012; Pergamon, Oxford, 1975).

3. C. W. Misner, K. S. Thorne, and J. A. Wheeler, Gravitation (W. H. Freeman, San Francisco, 1973).

4. J. P. A. Clark and D. M. Eardley, Astrophys. J. 215, 311 (1977).

5. A. V. Tutukov and L. R. Yungelson, Mon. Not. R. Astron. Soc. 260, 675 (1993).

6. V. M. Lipunov, K. A. Postnov, and M. E. Prokhorov, Astron. Lett. 23, 492 (1997).

7. B. P. Abbott, R. Abbott, T. D. Abbott, M. R. Abernathy, et al., Phys. Rev. Lett. 116, 241103 (2016).

8. B. P. Abbott, R. Abbott, T. D. Abbott, F. Acernese, et al., Phys. Rev. Lett. 118, 221101 (2017).

9. B. P. Abbott, R. Abbott, T. D. Abbott, F. Acernese, et al., Astrophys. J. Lett. 851, L35 (2017).

10. B. P. Abbott, R. Abbott, T. D. Abbott, F. Acernese, et al., Phys. Rev. Lett. 119, 141101 (2017).

11. B. P. Abbott, R. Abbott, T. D. Abbott, S. Abraham, et al., Phys. Rev. X 9, 031040 (2019).

12. D. V. Bisikalo, A. G. Zhilkin, and E. P. Kurbatov, Phys. Usp. 62, 1136 (2019).

13. D. V. Bisikalo and A. G. Zhilkin, Mon. Not. R. Astron. Soc. 494, 5520 (2020).

14. A. Peres, Phys. Rev. 128, 2471 (1962).

15. J. D. Bekenstein, Astrophys. J. 183, 657 (1973).

16. N. Bode and S. Phinney, in Proceedings of the APS April Meeting, April 14-17, 2007 (Am. Phys. Soc., Washington, DC, 2007), p. S1.010.

17. M. Megevand, M. Anderson, J. Frank, E. W. Hirschmann, L. Lehner, S. L. Liebling, P. M. Motl, and D. Neilsen, Phys. Rev. D 80, 024012 (2009).

18. B. Kocsis and A. Loeb, Phys. Rev. Lett. 101, 041101 (2008).

19. A. M. Cherepashchuk, Phys. Usp. 59, 910 (2016).

20. L. R. Corrales, Z. Haiman, and A. MacFadyen, Mon. Not. R. Astron. Soc. 404, 947 (2010).
21. M. J. Fitchett, Mon. Not. R. Astron. Soc. 203, 1049 (1983).

22. H. Pietilä, P. Heinamaki, S. Mikkola, and M. J. Valtonen, Celest. Mech. Dyn. Astron. 62, 377 (1995).

23. S. E. de Mink and A. King, Astrophys. J. Lett. 839, L7 (2017).

24. D. V. Bisikalo, A. G. Zhilkin, and E. P. Kurbatov, Astron. Rep. 63, 1 (2019).

25. F. Pretorius, Phys. Rev. Lett. 95, 121101 (2005).

26. M. Campanelli, C. O. Lousto, P. Marronetti, and Y. Zlochower, Phys. Rev. Lett. 96, 111101 (2006).

27. J. G. Baker, J. Centrella, Dae-Il Choi, M. Koppitz, and J. van Meter, Phys. Rev. Lett. 96, 111102 (2006).

28. M. I. Cohen, J. D. Kaplan, and M. A. Scheel, Phys. Rev. D 85, 024031 (2012).

29. P. Artymowicz and S. H. Lubow, Astrophys. J. 421, 651 (1994).

30. D. B. Bowen, V. Mewes, S. C. Noble, M. Avara, M. Campanelli, and J. H. Krolik, Astrophys. J. 879, 76 (2019).

31. P. V. Kaigorodov, D. V. Bisikalo, A. M. Fateeva, and A. Yu. Sytov, Astron. Rep. 54, 1078 (2010).

32. A. Yu. Sytov, P. V. Kaigorodov, A. M. Fateeva, and D. V. Bisikalo, Astron. Rep. 55, 793 (2011).

33. N. I. Shakura and R. A. Sunyaev, Astron. Astrophys. 24, 337 (1973).

34. V. M. Lipunov, G. Borner, and R. S. Wadhwa, Astrophysics of Neutron Stars (Springer, Berlin, 1992).

35. J. M. Bardeen, Nature (London, U.K.) 226, 64 (1970).

36. K. S. Thorne, Astrophys. J. 191, 507 (1974).

37. L.-X. Li and B. Paczynski, Astrophys. J. Lett. 534, L197 (2000).

38. A. A. Samarskii and I. P. Popov, Difference Methods for Solving Problems of Gas Dynamics, 2nd ed. (Nauka, Moscow, 1980) [in Russian].

39. D. V. Bisikalo and A. G. Zhilkin, Ann. Braz. Acad. Sci. 93 (Suppl. 1), e20200801 (2021).

40. L. I. Sedov, Similarity and Dimensional Methods in $\mathrm{Me}$ chanics (Mir, Moscow, 1982).

Translated by E. Petrova 